\title{
Approximate controllability of fractional impulsive partial neutral integrodifferential inclusions with infinite delay in Hilbert spaces
}

\section{Zuomao Yan ${ }^{*}$ and Xiumei Jia}

"Correspondence:

yanzuomao@163.com Department of Mathematics, Hexi University, Zhangye, Gansu 734000 P.R. China

\section{Springer}

\begin{abstract}
In this paper, the approximate controllability of fractional impulsive partial neutral integrodifferential inclusions with infinite delay in a Hilbert space is studied. By using the nonlinear alternative of Leray-Schauder type for multivalued maps due to O'Regan and properties of the $\alpha$-resolvent operator combined with approximation techniques, we derive a new set of sufficient conditions for the approximate controllability of fractional impulsive evolution system under the assumption that the corresponding linear system is approximately controllable. An example is provided to illustrate the obtained theory.
\end{abstract}

MSC: 34A37; 34K05; 93B05; 26A33

Keywords: approximate controllability; fractional impulsive partial neutral integrodifferential inclusions; multivalued map; infinite delay; $\alpha$-resolvent operator

\section{Introduction}

The study of impulsive functional differential equations is linked to their utility in simulating processes and phenomena subject to short-time perturbations during their evolution. The perturbations are performed discretely and their duration is negligible in comparison with the total duration of the processes and phenomena. One may refer to [1-3] and the references therein.

The nonlinear fractional differential equations has in recent years been an object of increasing interest because of its wide applicability in nonlinear oscillations of earthquakes, and many physical phenomena such as seepage flow in porous media and in fluid dynamic traffic models; see [4-6]. Recently, the existence of solutions for fractional semilinear differential or integrodifferential equations has been extensively studied by many authors [7-12]. Functional differential equations with infinite delay appear frequently in applications as model equations and for this reason the study of such equations has received great attention in the last few years. Many authors [13-15] were interested in the existence of solutions for fractional functional differential equations with infinite delay in Banach spaces. Further, the existence, uniqueness and other quantitative and qualitative properties of solutions to various fractional impulsive semilinear differential and integrodifferential systems have received considerable interest among researchers. With regard to this matter,

(c) 2015 Yan and Jia; licensee Springer. This article is distributed under the terms of the Creative Commons Attribution 4.0 International License (http://creativecommons.org/licenses/by/4.0/), which permits unrestricted use, distribution, and reproduction in any medium, provided you give appropriate credit to the original author(s) and the source, provide a link to the Creative Commons license, and indicate if changes were made. 
we refer the reader to Mophou [16], Shu et al. [17], Dabas and Chauhan [18], Balachandran et al. [19], and so on.

Controllability is one of the fundamental concepts in mathematical control theory and plays an important role in control systems. The exact controllability of nonlinear systems represented by fractional evolution equations or inclusions in abstract spaces has been considered in many publications; see [20-23]. There exist only a limited number of papers on the exact controllability of the fractional impulsive evolution systems. For example, Tai and Wang [24] studied the exact controllability of fractional-order impulsive neutral functional integrodifferential systems by using fractional calculus, a semigroup of operators and Krasnoselskii's fixed point theorem. Debbouche and Baleanu [25] established the exact controllability result of a class of fractional evolution nonlocal impulsive quasilinear delay integrodifferential systems. Liu and Li [26] discussed the exact controllability of impulsive fractional evolution inclusions in Banach spaces. However, in infinite-dimensional spaces the concept of exact controllability is usually too strong and, indeed, has limited applicability (see [27] and references therein). Approximate controllable systems are more prevalent and very often approximate controllability is completely adequate in applications (see $[28,29]$ and references therein). Therefore, it is important, in fact necessary, to study the weaker concept of controllability, namely approximate controllability for nonlinear systems. In recent years, for fractional semilinear control systems in Banach spaces, several papers were devoted to the approximate controllability. For example, Sakthivel et al. [30, 31] discussed the approximate controllability of semilinear fractional differential systems. The approximate controllability problem for nonlinear fractional stochastic system in Hilbert spaces has been investigated [32]. Kumar and Sukavanam [33] proved some sufficient conditions for the approximate controllability of fractional-order semilinear systems with bounded delay. Sukavanam and Kumar [34] discussed the approximate controllability of a fractional-order system in which the nonlinear term depends on both state and control variables. Yan [35] studied the approximate controllability of partial neutral functional differential systems of fractional order with state-dependent delay. Debbouche and Torres [36] derived the approximate controllability of a class of fractional nonlocal delay semilinear systems. It is well known that many control systems arising from realistic models can be described as partial fractional differential or integrodifferential inclusions (see $[23,26,37-39]$ and references therein), so it is natural to extend the concept of approximate controllability to dynamical systems represented by fractional impulsive differential or integrodifferential inclusions. In this paper, we consider the approximate controllability of a class of fractional impulsive partial neutral integrodifferential inclusions with infinite delay in Hilbert spaces of the form

$$
\begin{aligned}
& { }^{c} D^{\alpha} N\left(x_{t}\right) \in A N\left(x_{t}\right)+\int_{0}^{t} Q(t-s) N\left(x_{s}\right) d s+B u(s)+F\left(t, x_{t}, \int_{0}^{t} h\left(t, s, x_{s}\right) d s\right), \\
& \quad t \in J=[0, b], t \neq t_{k}, k=1, \ldots, m, \\
& x_{0}=\varphi \in \mathcal{B}, \quad x^{\prime}(0)=0, \\
& \Delta x\left(t_{k}\right)=I_{k}\left(x_{t_{k}}\right), \quad k=1, \ldots, m,
\end{aligned}
$$

where the unknown $x(\cdot)$ takes values in the Hilbert space $H$ with the norm $\|\cdot\|,{ }^{c} D^{\alpha}$ is the Caputo fractional derivative of order $\alpha \in(1,2), A,(Q(t))_{t \geq 0}$, are closed linear operators 
defined on a common domain which is dense in $(H,\|\cdot\|)$, the control function $u \in L^{2}(J, U)$, a Hilbert space of admissible control functions. Further, $B$ is a bounded linear operator from $U$ to $H$ and $D_{t}^{\alpha} \xi(t)$ represents the Caputo derivative of order $\alpha>0$ defined by

$$
D_{t}^{\alpha} \xi(t)=\int_{0}^{t} g_{n-\alpha}(t-s) \frac{d^{n}}{d s^{n}} \xi(s) d s
$$

where $n$ is the smallest integer greater than or equal to $\alpha$ and $g_{\beta}(t):=\frac{t^{\beta-1}}{\Gamma(\beta)}, t>0, \beta \geq 0$. The time history $x_{t}:(-\infty, 0] \rightarrow H$ given by $x_{t}(\theta)=x(t+\theta)$ belongs to some abstract phase space $\mathcal{B}$ defined axiomatically; and $F: J \times \mathcal{B} \times H \rightarrow \mathcal{P}(H)$ is a bounded, closed, convexvalued, multivalued map, $\mathcal{P}(H)$ is the family of all nonempty subsets of $H, G: J \times \mathcal{B} \rightarrow$ $H, N(\psi)=\psi(0)+G(t, \psi), \psi \in \mathcal{B}$, and $I_{k}: \mathcal{B} \rightarrow H(k=1, \ldots, m)$, are functions subject to some additional conditions. Moreover, let $0<t_{1}<\cdots<t_{m}<b$, are prefixed points and the symbol $\Delta x\left(t_{k}\right)=x\left(t_{k}^{+}\right)-x\left(t_{k}^{-}\right)$, where $x\left(t_{k}^{-}\right)$and $x\left(t_{k}^{+}\right)$represent the right and left limits of $x(t)$ at $t=t_{k}$, respectively.

To the best of our knowledge, there is no work reported on the approximate controllability of the fractional impulsive partial neutral integrodifferential inclusions with infinite delay, which is expressed in the form (1.1)-(1.3), and the aim of this paper is to close the gap. In this paper, motivated by the previously mentioned papers, we will study this interesting problem. Sufficient conditions for the existence are given by means of the nonlinear alternative of Leray-Schauder type for multivalued maps due to O'Regan [40] with the $\alpha$ resolvent operator combined with approximation techniques. Especially, the well-known results that appeared in [30-36] are generalized to the fractional multivalued settings and the case of infinite delay. Further, the operators $I_{k}(k=1, \ldots, m)$ are continuous but without imposing a completely continuous and Lipschitz condition. Therefore, our results have more applications in mathematical physical problems.

The rest of this paper is organized as follows. In Section 2, we introduce some notations and necessary preliminaries. In Section 3 we verify the existence of solutions for the fractional impulsive control system (1.1)-(1.3). In Section 4 we establish the approximate controllability of fractional impulsive control system (1.1)-(1.3). Finally in Section 5, an example is given to illustrate our results.

\section{Preliminaries}

In this section, we introduce some basic definitions, notations and lemmas which are used throughout this paper.

Let $(H,\|\cdot\|)$ be a Hilbert space. $C(J, H)$ is the Hilbert space of all continuous functions from $J$ into $H$ with the norm $\|x\|_{\infty}=\sup \{\|x(t)\|: t \in J\}$ and $L(H)$ denotes the Hilbert space of bounded linear operators from $H$ to $H$. A measurable function $x: J \rightarrow H$ is Bochner integrable if and only if $\|x\|$ is Lebesgue integrable. For properties of the Bochner integral see Yosida [41]. $L^{1}(J, H)$ denotes the Hilbert space of measurable functions $x: J \rightarrow H$ which are Bochner integrable normed by $\|x\|_{L^{1}}=\int_{0}^{b}\|x(t)\| d t$ for all $x \in L^{1}(J, H)$. Furthermore, the notation $B_{r}(x, H)$ stands for the closed ball with center at $x$ and radius $r>0$ in $H$.

Let $\mathcal{P}(H)$ denote the class of all nonempty subsets of $H$. Let $\mathcal{P}_{b d, c l}(H), \mathcal{P}_{c p, c v}(H)$, $\mathcal{P}_{b d, c l, c v}(H)$ and $\mathcal{P}_{c d}(H)$ denote respectively the families of all nonempty bounded-closed, compact-convex, bounded-closed-convex, and compact-acyclic (see [42]) subsets of $H$. For $x \in H$ and $Y, Z \in \mathcal{P}_{b d, c l}(H)$, we define $D(x, Y)=\inf \{\|x-y\|: y \in Y\}$ and $\tilde{\rho}(Y, Z)=$ 
$\sup _{a \in Y} D(a, Z)$, and the Hausdorff metric $H_{d}: \mathcal{P}_{b d, c l}(H) \times \mathcal{P}_{b d, c l}(H) \rightarrow \mathbb{R}^{+}$by $H_{d}(\tilde{A}, \tilde{B})=$ $\max \{\tilde{\rho}(\tilde{A}, \tilde{B}), \tilde{\rho}(\tilde{B}, \tilde{A})\}$.

$G$ is called upper semicontinuous (u.s.c.) on $H$ if, for each $x_{0} \in H$, the set $G\left(x_{0}\right)$ is a nonempty, closed subset of $H$ and if, for each open set $S$ of $H$ containing $G\left(x_{0}\right)$, there exists an open neighborhood $S$ of $x_{0}$ such that $G(S) \subseteq V$.F is said to be completely continuous if $G(V)$ is relatively compact, for every bounded subset $V \subseteq H$.

If the multivalued map $G$ is completely continuous with nonempty compact values, then $G$ is u.s.c. if and only if $F$ has a closed graph, i.e., $x_{n} \rightarrow x_{*}, y_{n} \rightarrow y_{*}, y_{n} \in G\left(x_{n}\right)$ imply $y_{*} \in G\left(x_{*}\right)$.

A multivalued map $G: J \rightarrow P_{b d, c l, c v}(H)$ is said to be measurable if for each $x \in H$, the function $t \mapsto D(x, G(t))$ is a measurable function on $J$.

Definition 2.1 Let $G: H \rightarrow P_{b d, c l}(H)$ be a multivalued map. Then $G$ is called a multivalued contraction if there exists a constant $\kappa \in(0,1)$ such that, for each $x, y \in H$, we have

$$
H_{d}(G(x)-G(y)) \leq \kappa\|x-y\|
$$

The constant $\kappa$ is called a contraction constant of $G$.

In this paper, we assume that the phase space $\left(\mathcal{B},\|\cdot\|_{\mathcal{B}}\right)$ is a semi-normed linear space of functions mapping $(-\infty, 0]$ into $H$, and satisfying the following fundamental axioms due to Hale and Kato (see, e.g., [43]).

(A) If $x:(-\infty, \sigma+b] \rightarrow H, b>0$, is such that $\left.x\right|_{[\sigma, \sigma+b]} \in C([\sigma, \sigma+b], H)$ and $x_{\sigma} \in \mathcal{B}$, then for every $t \in[\sigma, \sigma+b]$ the following conditions hold:

(i) $x_{t}$ is in $\mathcal{B}$;

(ii) $\|x(t)\| \leq \tilde{H}\left\|x_{t}\right\|_{\mathcal{B}}$;

(iii) $\left\|x_{t}\right\|_{\mathcal{B}} \leq K(t-\sigma) \sup \{\|x(s)\|: \sigma \leq s \leq t\}+M(t-\sigma)\left\|x_{\sigma}\right\|_{\mathcal{B}}$, where $\tilde{H} \geq 0$ is a constant; $K, M:[0, \infty) \rightarrow[1, \infty), K$ is continuous and $M$ is locally bounded; $\tilde{H}$, $K, M$ are independent of $x(\cdot)$.

(B) For the function $x(\cdot)$ in (A), the function $t \rightarrow x_{t}$ is continuous from $[\sigma, \sigma+b]$ into $\mathcal{B}$.

(C) The space $\mathcal{B}$ is complete.

Remark 2.1 ([3]) Let $\varphi \in \mathcal{B}$ and $t \leq 0$. The notation $\varphi_{t}$ represents the function defined by $\varphi_{t}=\varphi(t+\theta)$. Consequently, if the function $x(\cdot)$ in axiom (A) is such that $x_{0}=\varphi$, then $x_{t}=\varphi_{t}$. We observe that $\varphi_{t}$ is well defined for $t<0$ since the domain of $\varphi$ is $(-\infty, 0]$.

Remark 2.2 In the rest of this paper $M_{b}$ and $K_{b}$ are the constants defined by $M_{b}=$ $\sup _{t \in J} M(t)$ and $K_{b}=\sup _{t \in J} K(t)$.

To describe appropriately our problems we say that a function $x:[\mu, \tau] \rightarrow H$ is a normalized piecewise continuous function on $[\mu, \tau]$ if $x$ is piecewise continuous and continuous on $[\mu, \tau]$. We denote by $\mathcal{P C}([\mu, \tau], H)$ the space formed by the normalized piecewise continuous from $[\mu, \tau]$ into $H$. In particular, we introduce the space $\mathcal{P C}$ formed by all functions $x:[0, b] \rightarrow H$ such that $x$ is continuous at $t \neq t_{k}, x\left(t_{k}\right)=x\left(t_{k}^{-}\right)$and $x\left(t_{k}^{+}\right)$exist for $k=1,2, \ldots, m$. In this paper, we always assume that $\mathcal{P C}$ is endowed with the norm $\|x\|_{\mathcal{P C}}=\sup _{t \in[0, b]}\|x(t)\|$. Then $\left(\mathcal{P C},\|\cdot\|_{\mathcal{P C}}\right)$ is a Banach space. 
To simplify the notations, we put $t_{0}=0, t_{m+1}=b$ and for $x \in \mathcal{P C}$, we denote by $\hat{x}_{k} \in$ $C\left(\left[t_{k}, t_{k+1}\right] ; H\right), k=0,1, \ldots, m$, the function given by

$$
\hat{x}_{k}(t):= \begin{cases}x(t) & \text { for } t \in\left(t_{k}, t_{k+1}\right] \\ x\left(t_{k}^{+}\right) & \text {for } t=t_{k}\end{cases}
$$

Moreover, for $B \subseteq \mathcal{P C}$ we denote by $\hat{B_{k}}, k=0,1, \ldots, m$, the set $\hat{B}_{k}=\left\{\hat{x}_{k}: x \in B\right\}$.

Let us recall the following definitions and facts.

Definition 2.2 ([13]) A one-parameter family of bounded linear operators $\left(\mathcal{R}_{\alpha}(t)\right)_{t \geq 0}$ on $H$ is called an $\alpha$-resolvent operator for

$$
\begin{aligned}
& { }^{c} D^{\alpha} x(t)=A x(t)+\int_{0}^{t} Q(t-s) x(s) d s, \\
& x_{0}=\varphi \in H, \quad x^{\prime}(0)=0,
\end{aligned}
$$

if the following conditions are verified.

(a) The function $\mathcal{R}_{\alpha}(\cdot):[0, \infty) \rightarrow L(H)$ is strongly continuous and $\mathcal{R}_{\alpha}(0) x=x$ for all $x \in H$ and $\alpha \in(1,2)$.

(b) For $x \in D(A), \mathcal{R}_{\alpha}(\cdot) x \in C([0, \infty),[D(A)]) \cap C^{1}((0, \infty), H)$, and

$$
\begin{aligned}
& D_{t}^{\alpha} \mathcal{R}_{\alpha}(t) x=A \mathcal{R}_{\alpha}(t) x+\int_{0}^{t} Q(t-s) \mathcal{R}_{\alpha}(s) x d s \\
& D_{t}^{\alpha} \mathcal{R}_{\alpha}(t) x=\mathcal{R}_{\alpha}(t) A x+\int_{0}^{t} \mathcal{R}_{\alpha}(t-s) Q(s) x d s
\end{aligned}
$$

for every $t \geq 0$.

In this work we will consider the following conditions.

(P1) The operator $A: D(A) \subseteq H \rightarrow H$ is a closed linear operator with $[D(A)]$ dense in $H$. Let $\alpha \in(1,2)$. For some $\phi_{0} \in\left(0, \frac{\pi}{2}\right]$, for each $\phi<\phi_{0}$ there is a positive constant $C_{0}=C_{0}(\phi)$ such that $\lambda \in \rho(A)$ for each

$$
\lambda \in \Sigma_{0, \alpha \vartheta}=\{\lambda \in \mathbb{C}, \lambda \neq 0,|\arg (\lambda)|<\alpha \vartheta\}
$$

where $\vartheta=\phi+\frac{\pi}{2}$ and $\|R(\lambda, A)\| \leq \frac{C_{0}}{|\lambda|}$ for all $\lambda \in \Sigma_{0, \alpha \vartheta}$.

(P2) For all $t \geq 0, Q(t): D(Q(t)) \subseteq H \rightarrow H$ is a closed linear operator, $D(A) \subseteq D(Q(t))$ and $Q(\cdot) x$ is strongly measurable on $(0, \infty)$ for each $x \in D(A)$. There exists $b(\cdot) \in L_{\text {loc }}^{1}\left(\mathbb{R}^{+}\right)$such that $\widehat{b}(\lambda)$ exists for $\operatorname{Re}(\lambda)>0$ and $\|Q(t) x\| \leq b(t)\|x\|_{1}$ for all $t>0$ and $x \in D(A)$. Moreover, the operator-valued function $\widehat{Q}: \Sigma_{0, \pi / 2} \rightarrow L\left([D(A)], H\right.$ ) has an analytical extension (still denoted by $\widehat{Q}$ ) to $\Sigma_{0, \vartheta}$ such that $\|\widehat{Q}(\lambda) x\| \leq\|\widehat{Q}(\lambda)\|\|x\|_{1}$ for all $x \in D(A)$, and $\|\widehat{Q}(\lambda)\|=O\left(\frac{1}{|\lambda|}\right)$, as $|\lambda| \rightarrow \infty$.

(P3) There exists a subspace $D \subseteq D(A)$ dense in $[D(A)]$ and a positive constant $\widetilde{C}$ such that $A(D) \subseteq D(A), \widehat{Q}(\lambda)(D) \subseteq D(A)$, and $\|A \widehat{Q}(\lambda) x\| \leq \widetilde{C}\|x\|$ for every $x \in D$ and all $\lambda \in \Sigma_{0, \vartheta}$. 
In the sequel, for $r>0$ and $\theta \in\left(\frac{\pi}{2}, \vartheta\right)$,

$$
\Sigma_{r, \theta}=\{\lambda \in \mathbb{C},|\lambda|>r,|\arg (\lambda)|<\theta\},
$$

for $\Gamma_{r, \theta}, \Gamma_{r, \theta}^{i}, i=1,2,3$, are the paths

$$
\begin{aligned}
& \Gamma_{r, \theta}^{1}=\left\{t e^{i \theta}: t \geq r\right\}, \\
& \Gamma_{r, \theta}^{2}=\left\{t e^{i \xi}:|\xi| \leq \theta\right\}, \\
& \Gamma_{r, \theta}^{3}=\left\{t e^{-i \theta}: t \geq r\right\},
\end{aligned}
$$

and $\Gamma_{r, \theta}=\bigcup_{i=1}^{3} \Gamma_{r, \theta}^{i}$ oriented counterclockwise. In addition, $\rho_{\alpha}\left(G_{\alpha}\right)$ are the sets

$$
\rho_{\alpha}\left(G_{\alpha}\right)=\left\{\lambda \in \mathbb{C}: G_{\alpha}(\lambda):=\lambda^{\alpha-1}\left(\lambda^{\alpha} I-A-\widehat{Q}(\lambda)\right)^{-1} \in L(H)\right\} .
$$

We now define the operator family $\left(\mathcal{R}_{\alpha}(t)\right)_{t \geq 0}$ by

$$
\mathcal{R}_{\alpha}(t):= \begin{cases}\frac{1}{2 \pi i} \int_{\Gamma_{r, \theta}} e^{\lambda t} G_{\alpha}(\lambda) d \lambda, & t>0 \\ I, & t=0 .\end{cases}
$$

Lemma 2.1 ([13]) Assume that conditions (P1)-(P3) are fulfilled. Then there exists a unique $\alpha$-resolvent operator for problem (2.1)-(2.2).

Lemma 2.2 ([13]) The function $\mathcal{R}_{\alpha}:[0, \infty) \rightarrow L(H)$ is strongly continuous and $\mathcal{R}_{\alpha}:$ $(0, \infty) \rightarrow L(H)$ is uniformly continuous.

Definition 2.3 ([13]) Let $\alpha \in(1,2)$, we define the family $\left(\mathcal{S}_{\alpha}(t)\right)_{t \geq 0}$ by

$$
\mathcal{S}_{\alpha}(t) x:=\int_{0}^{t} g_{\alpha-1}(t-s) \mathcal{R}_{\alpha}(s) d s
$$

for each $t \geq 0$.

Lemma 2.3 ([13]) If the function $\mathcal{R}_{\alpha}(\cdot)$ is exponentially bounded in $L(H)$, then $\mathcal{S}_{\alpha}(\cdot)$ is exponentially bounded in $L(H)$.

Lemma $2.4([13])$ If the function $\mathcal{R}_{\alpha}(\cdot)$ is exponentially bounded in $L([D(A)])$, then $\mathcal{S}_{\alpha}(\cdot)$ is exponentially bounded in $L([D(A)])$.

Lemma 2.5 ([13]) If $R\left(\lambda_{0}^{\alpha}, A\right)$ is compact for some $\lambda_{0}^{\alpha} \in \rho(A)$, then $\mathcal{R}_{\alpha}(t)$ and $\mathcal{S}_{\alpha}(t)$ are compact for all $t>0$.

Let $x_{b}\left(x_{0} ; u\right)$ be the state value of system (1.1)-(1.3) at terminal time $b$ corresponding to the control $u$ and the initial value $x_{0}=\varphi \in \mathcal{B}$. Introduce the set

$$
\mathcal{B}\left(b, x_{0}\right)=\left\{x_{b}\left(x_{0} ; u\right)(0): u(\cdot) \in L^{2}(J, U)\right\}
$$

which is called the reachable set of system (1.1)-(1.3) at terminal time $b$, its closure in $H$ is denoted by $\overline{\mathcal{B}\left(b, x_{0}\right)}$. 
Definition 2.4 A function $x:(-\infty, b] \rightarrow H$ is called a mild solution of problem (1.1)-(1.3) if $x_{0}=\varphi \in \mathcal{B}$ for every $s \in J$ and $\Delta x\left(t_{k}\right)=I_{k}\left(x_{t_{k}}\right), k=1, \ldots, m$, the restriction of $x(\cdot)$ to the interval $\left(t_{k}, t_{k+1}\right](k=0,1, \ldots, m)$ is continuous, and

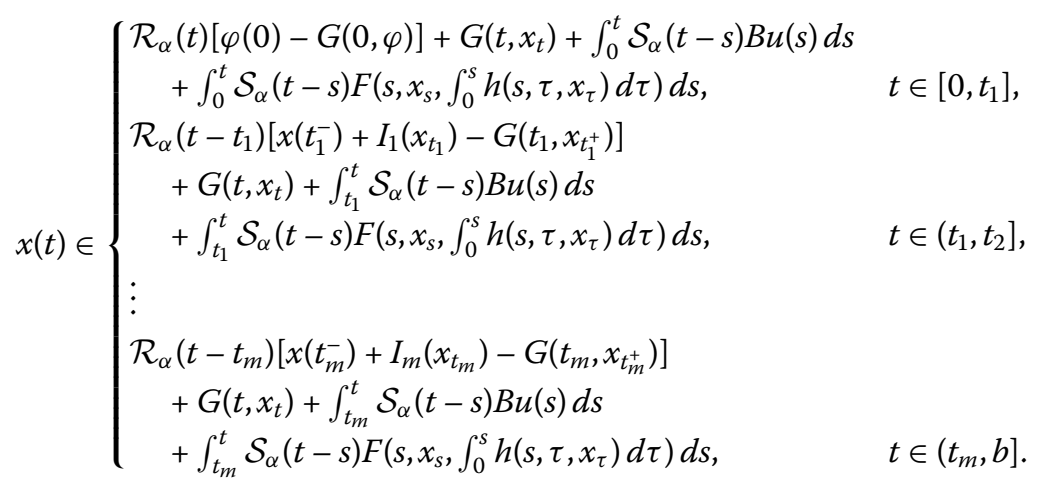

Definition 2.5 System (1.1)-(1.3) is said to be approximately controllable on the interval $[0, b]$ if $\overline{\mathcal{B}\left(b, x_{0}\right)}=H$ that is, if it is possible to steer the system from the initial point $x_{0}$ to within a distance $\varepsilon>0$ from all the final points in the state space $H$ at time $b$.

It is convenient at this point to define operators

$$
\begin{aligned}
& \Gamma_{\tau}^{b}=\int_{\tau}^{b} \mathcal{S}_{\alpha}(b-s) B B^{*} \mathcal{S}_{\alpha}^{*}(b-s) d s, \quad 0 \leq \tau<b, \\
& \Gamma_{t_{k-1}}^{t_{k}}=\int_{t_{k-1}}^{t_{k}} \mathcal{S}_{\alpha}\left(t_{k}-s\right) B B^{*} \mathcal{S}_{\alpha}^{*}\left(t_{k}-s\right) d s, \quad k=1,2, \ldots, m, m+1, \\
& R\left(a, \Gamma_{t_{k-1}}^{t_{k}}\right)=\left(a I+\Gamma_{t_{k-1}}^{t_{k}}\right)^{-1} \quad \text { for } a>0, k=1,2, \ldots, m, m+1,
\end{aligned}
$$

where $B^{*}$ denotes the adjoint of $B$ and $\mathcal{S}_{\alpha}^{*}(t)$ is the adjoint of $\mathcal{S}_{\alpha}(t)$. It is straightforward that the operator $\Gamma_{t_{k-1}}^{t_{k}}$ is a linear bounded operator.

Lemma 2.6 The linear integrodifferential Cauchy problem corresponding to system (1.1)(1.3) is approximately controllable on $J$ if and only if $a R\left(a, \Gamma_{\tau}^{s}\right) \rightarrow 0,0 \leq \tau<s \leq b$, as $a \rightarrow$ $0^{+}$in the strong operator topology.

The proof of this lemma is a straightforward adaptation of the proof of [28], Theorem 2 .

Lemma 2.7 $A$ set $B \subseteq \mathcal{P C}$ is relatively compact in $\mathcal{P C}$ if and only if the set $\hat{B}_{k}$ is relatively compact in $C\left(\left[t_{k}, t_{k+1}\right] ; H\right)$ for every $k=0,1, \ldots, m$.

Lemma 2.8 ([40] Nonlinear alternative of Leray-Schauder type for multivalued maps due to O'Regan) Let $H$ be a Hilbert space with $V$ an open, convex subset of $H$ and $y \in H$. Suppose

(a) $\Phi: \bar{V} \rightarrow \mathcal{P}_{c d}(H)$ has a closed graph, and

(b) $\Phi: \bar{V} \rightarrow \mathcal{P}_{c d}(H)$ is a condensing map with $\Phi(\bar{V})$ a subset of a bounded set in $H$ hold.

Then either

(i) $\Phi$ has a fixed point in $\bar{V}$; or

(ii) there exist $y \in \partial V$ and $\lambda \in(0,1)$ with $y \in \lambda \Phi(y)+(1-\lambda)\left\{y_{0}\right\}$. 


\section{Existence of solutions for fractional impulsive control systems}

In this section, we prove the existence of solutions for the fractional impulsive control system (1.1)-(1.3). We make the following hypotheses.

(H1) The operator families $\mathcal{R}_{\alpha}(t)$ and $\mathcal{S}_{\alpha}(t)$ are compact for all $t>0$, and there exist constants $M$ and $\delta$ such that $\left\|\mathcal{R}_{\alpha}(t)\right\|_{L(H)} \leq M e^{\delta t}$ and $\left\|\mathcal{S}_{\alpha}(t)\right\|_{L(H)} \leq M e^{\delta t}$ for every $t \in J$.

(H2) The function $G: J \times \mathcal{B} \rightarrow H$ is continuous and there exists a $L>0$ such that

$$
\left\|G\left(t, \psi_{1}\right)-G\left(t, \psi_{2}\right)\right\| \leq L\left[\left|t_{1}-t_{2}\right|+\left\|\psi_{1}-\psi_{2}\right\|_{\mathcal{B}}\right], \quad t_{1}, t_{2} \in J, \psi_{1}, \psi_{2} \in \mathcal{B},
$$

and

$$
\|G(t, \psi)\| \leq L\left(\|\psi\|_{\mathcal{B}}+1\right), \quad t \in J, \psi \in \mathcal{B} .
$$

(H3) (i) For each $(t, s) \in \Lambda$ the function $h(t, s, \cdot): \mathcal{B} \rightarrow H$ is continuous and for each $x \in \mathcal{B}$, the function $h(\cdot, \cdot, x): \Lambda \rightarrow H$ is strongly measurable.

(ii) There exists a continuous function $p: \Lambda \rightarrow[0, \infty)$, such that

$$
\|h(t, s, \psi)\| \leq p(t, s) \Theta_{0}\left(\|\psi\|_{\mathcal{B}}\right)
$$

for a.e. $t, s \in J, \psi \in \mathcal{B}$, where $\Theta_{0}:[0, \infty) \rightarrow(0, \infty)$ is a continuous nondecreasing function.

(H4) The multivalued map $F: J \times \mathcal{B} \times H \rightarrow \mathcal{P}_{b d, c l, c v}(H)$; for each $t \in J$, the function $F(t, \cdot, \cdot): \mathcal{B} \times H \rightarrow \mathcal{P}_{b d, c l, c v}(H)$ is u.s.c. and for each $(\psi, y) \in \mathcal{B} \times H$, the function $F(\cdot, \psi, y)$ is measurable; for each fixed $(\psi, y) \in \mathcal{B} \times H$, the set

$$
S_{F, \psi}=\left\{f \in L^{1}(J, H): f(t) \in F\left(t, \psi, \int_{0}^{t} h(t, s, \psi) d s\right) \text { for a.e. } t \in J\right\}
$$

is nonempty.

(H5) There exist a continuous function $m: J \rightarrow[0, \infty)$ and a continuous nondecreasing function $\Theta:[0, \infty) \rightarrow(0, \infty)$ such that

$$
\|F(t, \psi, y)\|=\sup \{\|f\|: f \in F(t, \psi, y)\} \leq m(t) \Theta\left(\|\psi\|_{\mathcal{B}}+\|y\|\right),
$$

for a.e. $t \in J$ and each $\psi \in \mathcal{B}, y \in H$ with

$$
\int_{1}^{\infty} \frac{1}{s+\Theta(s)+\Theta_{0}(s)} d s=\infty
$$

(H6) The functions $I_{k}: \mathcal{B} \rightarrow H$ are continuous and there exist constants $c_{k}$ such that

$$
\limsup _{\|\psi\|_{\mathcal{B}} \rightarrow \infty} \frac{\left\|I_{k}(\psi)\right\|}{\|\psi\|_{\mathcal{B}}}=c_{k}
$$

for every $\psi \in \mathcal{B}, k=1, \ldots, m$. 
Lemma 3.1 ([44]) Let J be a compact interval and $H$ be a Hilbert space. Let $F$ be a multivalued map satisfying $(\mathrm{H} 4)$ and let $P$ be a linear continuous operator from $L^{1}(J, H)$ to $C(J, H)$. Then the operator

$$
P \circ S_{F}: C(J, H) \rightarrow \mathcal{P}_{c p, c v}(H), \quad x \rightarrow\left(P \circ S_{F}\right)(x):=P\left(S_{F}, x\right)
$$

is a closed graph in $C(J, H) \times C(J, H)$.

Theorem 3.1 If assumptions (H1)-(H6) are satisfied. Further, suppose that, for all $a>0$, then system (1.1)-(1.3) has at least one mild solution on J, provided that

$$
\max _{1 \leq k \leq m}\left\{M_{2}\left[1+K_{b}\left(M c_{k}+M L\right)\right]+M_{3} K_{b} M L\right\}<1,
$$

where $M_{2}=M N_{*}\left(1+\frac{1}{a} M_{*}^{2} N_{*}^{2} M_{1}^{2} b\right), M_{3}=\left(1+\frac{1}{a} M M_{*} N_{*} M_{1}^{2} b\right) N_{*}, M_{*}=M \max \left\{1, e^{\delta b}\right\}, N_{*}=$ $\max \left\{1, e^{-\delta b}\right\}, M_{1}=\|B\|$.

Proof Consider the space $\mathcal{Y}=\left\{x:(-\infty, b] \rightarrow H ; x_{0}=0,\left.x\right|_{J} \in \mathcal{P C}(J, H)\right\}$ endowed with the uniform convergence topology and define the multivalued map $\Phi: \mathcal{Y} \rightarrow \mathcal{P}(\mathcal{Y})$ by $\Phi x$, the set of $\rho \in \mathcal{Y}$ such that

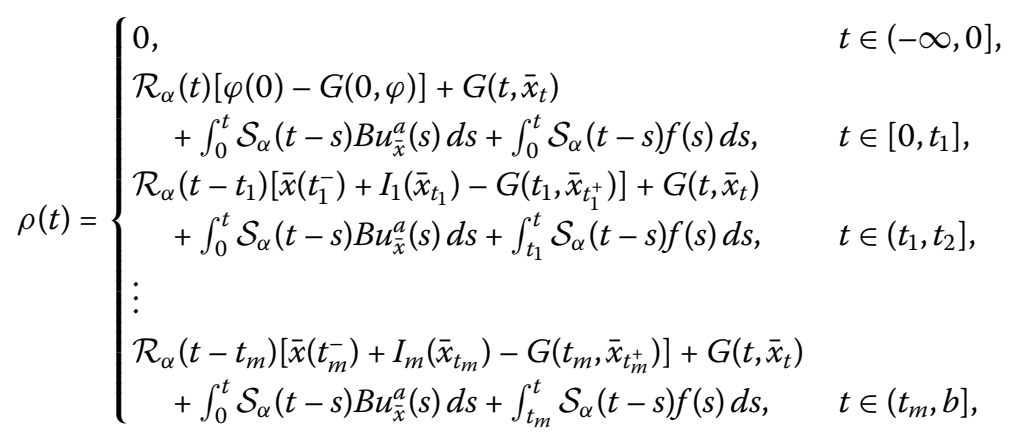

where

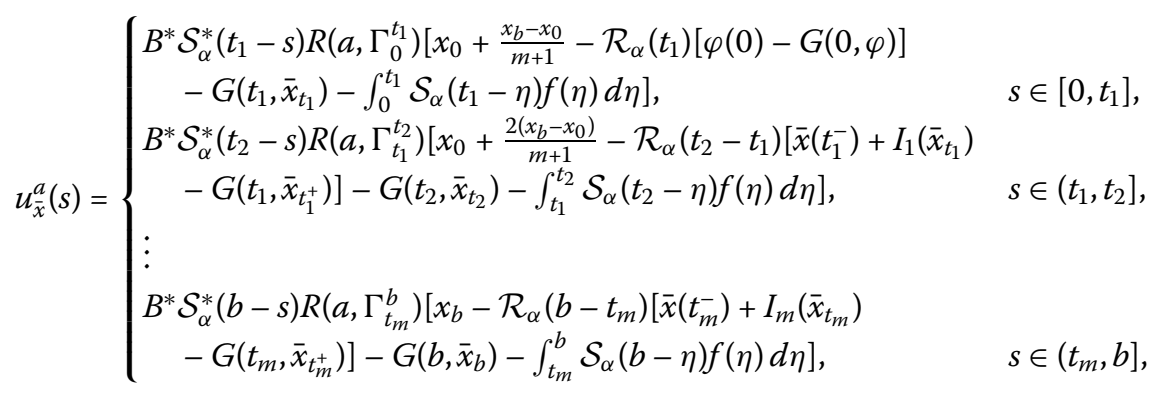

and $f \in S_{F, \bar{x}}=\left\{f \in L^{1}(J, H): f(t) \in F\left(t, \bar{x}_{t}, \int_{0}^{t} h\left(t, s, \bar{x}_{s}\right) d s\right)\right.$ a.e. $\left.t \in J\right\}$, and $\bar{x}:(-\infty, 0] \rightarrow H$ is such that $\bar{x}_{0}=\varphi$ and $\bar{x}=x$ on $J$. In what follows, we aim to show that the operator $\Phi$ has a fixed point, which is a solution of problem (1.1)-(1.3). 
Let $\left\{\sigma_{n}: n \in \mathbb{N}\right\}$ be a decreasing sequence in $\left(0, t_{1}\right) \subset(0, b)$ such that $\lim _{n \rightarrow \infty} \sigma_{n}=0$. To prove the above theorem, we consider the following problem:

$$
\begin{aligned}
& { }^{c} D^{\alpha} \tilde{N}\left(x_{t}\right) \in A \widetilde{N}\left(x_{t}\right)+\int_{0}^{t} Q(t-s) \tilde{N}\left(x_{s}\right) d s+B u(s)+F\left(t, x_{t}, \int_{0}^{t} h\left(t, s, x_{s}\right) d s\right), \\
& t \in J=[0, b], t \neq t_{k}, k=1, \ldots, m, \\
& x_{0}=\varphi \in \mathcal{B}, \quad x^{\prime}(0)=0, \\
& \Delta x\left(t_{k}\right)=\mathcal{R}_{\alpha}\left(\sigma_{n}\right) I_{k}\left(x_{t_{k}}\right), \quad k=1, \ldots, m,
\end{aligned}
$$

where $\tilde{N}\left(x_{t}\right)=\varphi(0)+\mathcal{R}_{\alpha}\left(\sigma_{n}\right) G\left(t, x_{t}\right)$. We shall show that the problem has at least one mild solution $x_{n} \in \mathcal{Y}$.

For fixed $n \in \mathbb{N}$, set the multivalued map $\Phi_{n}: \mathcal{Y} \rightarrow \mathcal{P}(\mathcal{Y})$ by $\Phi_{n} x$ the set of $\rho_{n} \in \mathcal{Y}$ such that

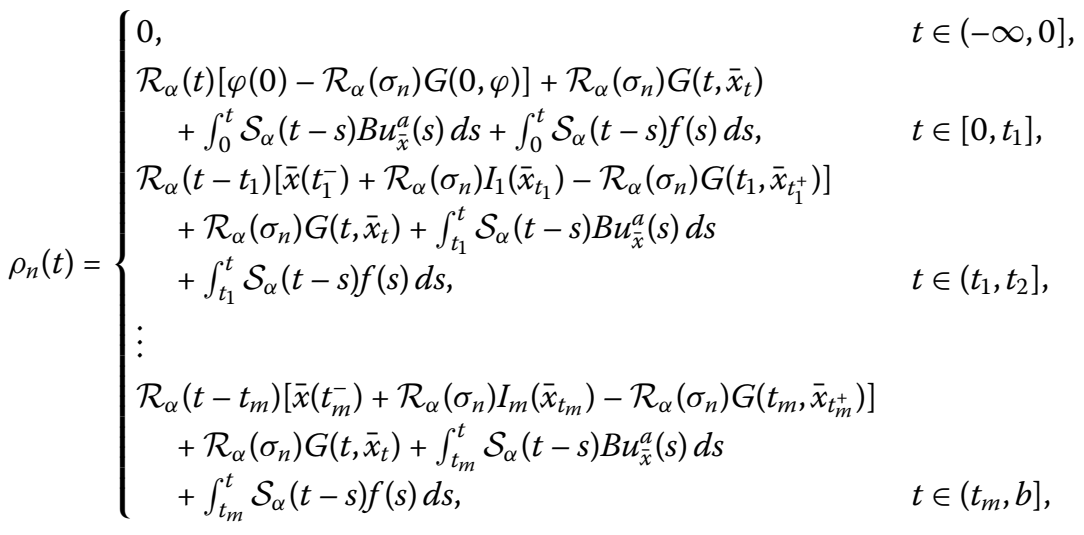

where

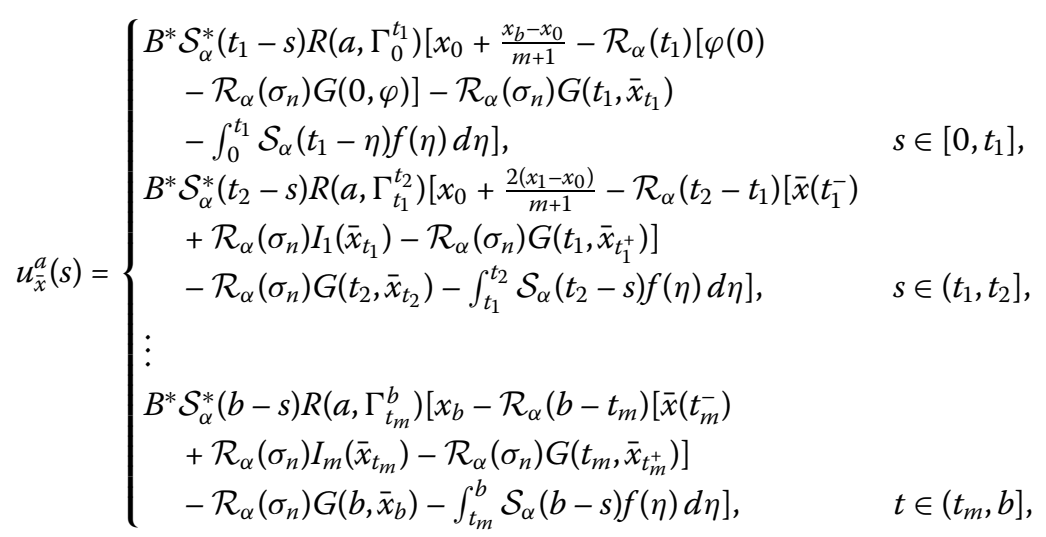

and $f \in S_{F, \bar{x}}$. It is easy to see that the fixed point of $\Phi_{n}$ is a mild solution of problem (3.3)(3.5).

Step 1 . We shall show there exists an open set $V \subseteq \mathcal{Y}$ with $x \in \lambda \Phi_{n} x$ for $\lambda \in(0,1)$ and $x \notin \partial V$. 
Let $\lambda \in(0,1)$ and let $x \in \lambda \Phi_{n} x$, then there exists an $f \in S_{F, \bar{x}}$ such that we have

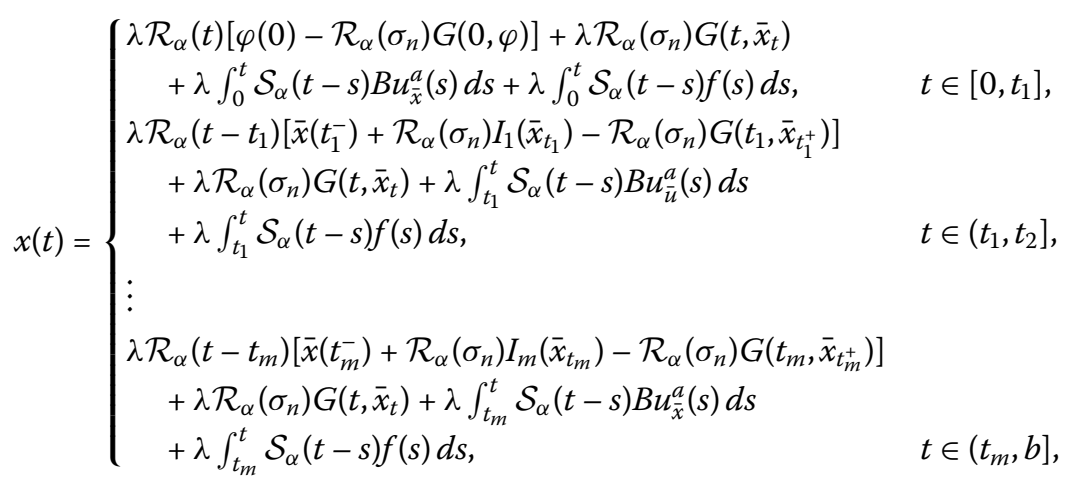

for some $\lambda \in(0,1)$. On the other hand, from condition (H6), we conclude that there exist positive constants $\epsilon_{k}(k=1, \ldots, m), \gamma_{1}$ such that, for all $\|\psi\|_{\mathcal{B}}>\gamma_{1}$,

$$
\begin{aligned}
& \left\|I_{k}(\psi)\right\| \leq\left(c_{k}+\epsilon_{k}\right)\|\psi\|_{\mathcal{B}}, \\
& \max _{1 \leq k \leq m}\left\{M_{2}\left[1+K_{b}\left(M\left(c_{k}+\epsilon_{k}\right)+M L\right)\right]+M_{3} K_{b} M L\right\}<1 .
\end{aligned}
$$

Let

$$
F_{1}=\left\{\psi:\|\psi\|_{\mathcal{B}} \leq \gamma_{1}\right\}, \quad F_{2}=\left\{\psi:\|\psi\|_{\mathcal{B}}>\gamma_{1}\right\}, \quad C_{1}=\max \left\{\left\|I_{k}(\psi)\right\|, x \in F_{1}\right\} .
$$

Therefore

$$
\left\|I_{k}(\psi)\right\| \leq C_{1}+\left(c_{k}+\epsilon_{k}\right)\|\psi\|_{\mathcal{B}}
$$

Then, by (H1)-(H5) and (3.7), from the above equation, we have for $t \in\left[0, t_{1}\right]$

$$
\begin{aligned}
\|x(t)\| \leq & M e^{\delta t}\left[\tilde{H}\|\varphi\|_{\mathcal{B}}+M e^{\delta \sigma_{n}} L\left(\|\varphi\|_{\mathcal{B}}+1\right)\right]+M e^{\delta \sigma_{n}} L\left(\left\|\bar{x}_{t}\right\|_{\mathcal{B}}+1\right) \\
& +M e^{\delta t} \frac{1}{a} M e^{\delta b} N_{*}^{2} t_{1} M_{1}^{2} M e^{\delta \sigma_{n}} L\left(\left\|\bar{x}_{t_{1}}\right\|_{\mathcal{B}}+1\right)+M e^{\delta t} \frac{1}{a} M e^{\delta b} N_{*}^{2} t_{1} M_{1}^{2} \tilde{N}_{0} \\
& +M e^{\delta t} \int_{0}^{t} e^{-\delta s} m(s) \Theta\left(\left\|\bar{x}_{s}\right\|_{\mathcal{B}}+\int_{0}^{s} p(s, \tau) \Theta_{0}\left(\left\|\bar{x}_{\tau}\right\|_{\mathcal{B}}\right) d \tau\right) d s,
\end{aligned}
$$

where

$$
\begin{aligned}
\tilde{N}_{0}= & \left\|x_{0}\right\|+\left\|\frac{x_{1}-x_{0}}{m+1}\right\|+M_{*}\left[\|\varphi(0)\|+M e^{\delta \sigma_{n}} L\left(\|\varphi\|_{\mathcal{B}}+1\right)\right] \\
& +M_{*} \int_{0}^{t_{1}} e^{-\delta \eta} m_{f}(\eta) \Theta\left(\left\|\bar{x}_{\eta}\right\|_{\mathcal{B}}+\int_{0}^{\eta} p(\eta, \vartheta) \Theta_{0}\left(\left\|\bar{x}_{\vartheta}\right\|_{\mathcal{B}}\right) d \vartheta\right) d \eta, \quad M_{1}=\|B\| .
\end{aligned}
$$

Similarly, for any $t \in\left(t_{k}, t_{k+1}\right], k=1, \ldots, m$, we have

$$
\begin{aligned}
\|x(t)\| \leq & M e^{\delta\left(t-t_{k}\right)}\left\{\left\|\bar{x}\left(t_{k}^{-}\right)\right\|+M e^{\delta \sigma_{n}}\left[C_{1}+\left(c_{k}+\epsilon_{k}\right)\left\|\bar{x}_{t_{k}}\right\|_{\mathcal{B}}\right]+M e^{\delta \sigma_{n}} L\left(\left\|\bar{x}_{t_{k}^{+}}\right\|_{\mathcal{B}}+1\right)\right\} \\
& +M e^{\delta \sigma_{n}} L\left(\left\|\bar{x}_{t}\right\|_{\mathcal{B}}+1\right)+M e^{\delta t} \frac{1}{a} M e^{\delta b} N_{*}^{2} M_{1}^{2}\left(t_{k+1}-t_{k}\right)\left\{M _ { * } N _ { * } \left[\left\|\bar{x}\left(t_{k}^{-}\right)\right\|\right.\right.
\end{aligned}
$$




$$
\begin{aligned}
& \left.+M e^{\delta \sigma_{n}}\left[C_{1}+\left(c_{k}+\epsilon_{k}\right)\left\|\bar{x}_{t_{k}}\right\|_{\mathcal{B}}\right]+M e^{\delta \sigma_{n}} L\left(\left\|\bar{x}_{t_{k}^{+}}\right\|_{\mathcal{B}}+1\right)\right] \\
& \left.+M e^{\delta \sigma_{n}} L\left(\left\|\bar{x}_{t_{k+1}}\right\|_{\mathcal{B}}+1\right)\right\}+M e^{\delta t} \frac{1}{a} M e^{\delta b} N_{*}^{2} M_{1}^{2}\left(t_{k+1}-t_{k}\right) \tilde{N}_{k} \\
& +M e^{\delta t} \int_{t_{k}}^{t} e^{-\delta s} m(s) \Theta\left(\left\|\bar{x}_{t}\right\|_{\mathcal{B}}+\int_{0}^{s} p(s, \tau) \Theta_{0}\left(\left\|\bar{x}_{\tau}\right\|_{\mathcal{B}}\right) d \tau\right) d s
\end{aligned}
$$

where

$$
\begin{aligned}
\tilde{N}_{k}= & \left\|x_{0}\right\|+\left\|\frac{(k+1)\left(x_{1}-x_{0}\right)}{m+1}\right\| \\
& +M_{*} \int_{t_{k}}^{t_{k+1}} e^{-\delta \eta} m_{f}(\eta) \Theta\left(\left\|\bar{x}_{\eta}\right\|_{\mathcal{B}}+\int_{0}^{\eta} p(\eta, \vartheta) \Theta_{0}\left(\left\|\bar{x}_{\vartheta}\right\|_{\mathcal{B}}\right) d \vartheta\right) d \eta .
\end{aligned}
$$

Then, for all $t \in[0, b]$, we have

$$
\begin{aligned}
\|x(t)\| \leq & \tilde{M} e^{\delta t}+M e^{\delta t} N_{*}\left(1+\frac{1}{a} M_{*}^{2} N_{*}^{2} M_{1}^{2} b\right)\left[\left\|\bar{x}\left(t_{k}^{-}\right)\right\|+M e^{\delta \sigma_{n}}\left(c_{k}+\epsilon_{k}\right)\left\|\bar{x}_{t_{k}}\right\|_{\mathcal{B}}\right. \\
& \left.+M e^{\delta \sigma_{n}} L\left\|\bar{x}_{t_{k}^{+}}\right\|_{\mathcal{B}}\right]+M e^{\delta t} \frac{1}{a} M_{*} N_{*}^{2} M_{1}^{2} b M e^{\delta \sigma_{n}} L\left\|\bar{x}_{t_{k+1}}\right\|_{\mathcal{B}}+M e^{\delta \sigma_{n}} L\left\|\bar{x}_{t}\right\|_{\mathcal{B}} \\
& +M e^{\delta t} \int_{0}^{t} e^{-\delta s} m(s) \Theta\left(\left\|\bar{x}_{s}\right\|_{\mathcal{B}}+\int_{0}^{s} p(s, \tau) \Theta_{0}\left(\left\|\bar{x}_{\tau}\right\|_{\mathcal{B}}\right) d \tau\right) d s,
\end{aligned}
$$

where

$$
\begin{aligned}
\tilde{M}= & \max \left\{M\left[\tilde{H}\|\varphi\|_{\mathcal{B}}+M e^{\delta \sigma_{n}} L\left(\|\varphi\|_{\mathcal{B}}+1\right)\right]+M e^{\delta \sigma_{n}} L\right. \\
& +M \frac{1}{a} M_{*} N_{*}^{2} t_{1} M_{1}^{2} M e^{\delta \sigma_{n}} L+M \frac{1}{a} M_{*} N_{*}^{2} t_{1} \widetilde{N}_{0}, \\
& M N_{*}\left(1+\frac{1}{a} M_{*}^{2} N_{*}^{2} M_{1}^{2} b\right)\left[M e^{\delta \sigma_{n}} C_{1}+M e^{\delta \sigma_{n}} L\right]+M e^{\delta \sigma_{n}} L \\
& \left.+M \frac{1}{a} M_{*} N_{*}^{2} M_{1}^{2} b M e^{\delta \sigma_{n}} L+M \frac{1}{a} M_{*} N_{*}^{2} M_{1}^{2} b \tilde{N}\right\}, \quad \widetilde{N}=\max _{1 \leq k \leq m}\left\{\tilde{N}_{k}\right\} .
\end{aligned}
$$

Since $\lim _{n \rightarrow \infty} \sigma_{n}=0$, it follows that

$$
\begin{aligned}
\|x(t)\| \leq & \tilde{M} e^{\delta t}+M e^{\delta t} N_{*}\left(1+\frac{1}{a} M_{*}^{2} N_{*}^{2} M_{1}^{2} b\right)\left[\left\|\bar{x}\left(t_{k}^{-}\right)\right\|+M\left(c_{k}+\epsilon_{k}\right)\left\|\bar{x}_{t_{k}}\right\|_{\mathcal{B}}\right. \\
& \left.+M L\left\|\bar{x}_{t_{k}^{+}}\right\|_{\mathcal{B}}\right]+M e^{\delta t} \frac{1}{a} M_{*} N_{*}^{2} M_{1}^{2} b M L\left\|\bar{x}_{t_{k+1}}\right\|_{\mathcal{B}}+M L\left\|\bar{x}_{t}\right\|_{\mathcal{B}} \\
& +M e^{\delta t} \int_{0}^{t} e^{-\delta s} m(s) \Theta\left(\left\|\bar{x}_{s}\right\|_{\mathcal{B}}+\int_{0}^{s} p(s, \tau) \Theta_{0}\left(\left\|\bar{x}_{\tau}\right\|_{\mathcal{B}}\right) d \tau\right) d s .
\end{aligned}
$$

It is easy to see that

$$
\left\|\bar{x}_{t}\right\|_{\mathcal{B}} \leq M_{b}\|\varphi\|_{\mathcal{B}}+K_{b}\|x\|_{t}, \quad t \in[0, b],
$$

where $\|x\|_{t}=\sup _{0 \leq s \leq t}\|x(s)\|$. If $v(t)=M_{b}\|\varphi\|_{\mathcal{B}}+K_{b}\|x\|_{t}, t \in[0, b]$, we obtain 


$$
\begin{aligned}
v(t) \leq & M_{b}\|\varphi\|_{\mathcal{B}}+\widetilde{M} e^{\delta t}+M e^{\delta t} N_{*}\left(1+\frac{1}{a} M_{*}^{2} N_{*}^{2} M_{1}^{2} b\right) v(t) \\
& +K_{b} M e^{\delta t} N_{*}\left(1+\frac{1}{a} M_{*}^{2} N_{*}^{2} M_{1}^{2} b\right)\left[M\left(c_{k}+\epsilon_{k}\right) v(t)+M L v(t)\right] \\
& +K_{b} M e^{\delta t} \frac{1}{a} M_{*} N_{*}^{2} M_{1}^{2} b M L v(t)+K_{b} M L v(t) \\
& +K_{b} M e^{\delta t} \int_{0}^{t} e^{-\delta s} m(s) \Theta\left(v(s)+\int_{0}^{s} p(s, \tau) \Theta_{0}(v(\tau)) d \tau\right) d s .
\end{aligned}
$$

Since $\widetilde{L}=\max _{1 \leq k \leq m}\left\{M_{2}\left[1+K_{b}\left(M\left(c_{k}+\epsilon_{k}\right)+M L\right)\right]+M_{3} K_{b} M L\right\}<1$, we obtain

$$
\begin{aligned}
e^{-\delta t} v(t) \leq & \frac{1}{1-\widetilde{L}}\left[N_{*} M_{b}\|\varphi\|_{\mathcal{B}}+\tilde{M}\right. \\
& \left.+K_{b} M \int_{0}^{t} e^{-\delta s} m(s) \Theta\left(v(s)+\int_{0}^{s} p(s, \tau) \Theta_{0}(v(\tau)) d \tau\right) d s\right] .
\end{aligned}
$$

Denoting by $w(t)$ the right-hand side of the above inequality, we have

$$
v(t) \leq e^{\delta t} w(t) \quad \text { for all } t \in J
$$

and

$$
\begin{aligned}
w(0) & =\frac{1}{1-\widetilde{L}}\left[N_{*} M_{b}\|\varphi\|_{\mathcal{B}}+\widetilde{M}\right], \\
w^{\prime}(t) & =\frac{1}{1-\widetilde{L}} K_{b} M e^{-\delta t} m(t) \Theta\left(v(t)+\int_{0}^{t} p(t, s) \Theta_{0}(v(s)) d s\right) \\
& \leq \frac{1}{1-\widetilde{L}} K_{b} M e^{-\delta t} m(t) \Theta\left(e^{\delta t} w(t)+\int_{0}^{t} p(t, s) \Theta_{0}\left(e^{\delta s} w(s)\right) d s\right), \quad t \in J .
\end{aligned}
$$

Let $\xi(t)=e^{\delta t} w(t)+\int_{0}^{t} p(t, s) \Theta_{0}\left(e^{\delta s} w(s)\right) d s$, then $\xi(0)=w(0), e^{\delta t} w(t) \leq \xi(t)$, and for each $t \in J$ we have

$$
\begin{aligned}
(\xi(t))^{\prime} & =\delta e^{\delta t} w(t)+w^{\prime}(t) e^{\delta t}+p(t, t) \Theta_{0}\left(e^{\delta t} w(t)\right) \\
& \leq \delta \xi(t)+\frac{1}{1-\widetilde{L}} K_{b} M m(t) \Theta(\xi(t))+p(t, t) \Theta_{0}(\xi(t)) \\
& \leq \max \left\{\delta, \frac{1}{1-\widetilde{L}} K_{b} M m(t), p(t, t)\right\}\left[\xi(t)+\Theta(\xi(t))+\Theta_{0}(\xi(t))\right], \quad t \in J .
\end{aligned}
$$

This implies that

$$
\int_{\xi(0)}^{\xi(t)} \frac{d \zeta}{\zeta+\Theta(\varsigma)+\Theta_{0}(\varsigma)} \leq \int_{0}^{b} \max \left\{\delta, \frac{1}{1-\widetilde{L}} K_{b} M m(t), p(t, t)\right\} d t<\infty
$$

This inequality shows that there is a constant $\widetilde{K}$ such that $\xi(t) \leq \widetilde{K}, t \in J$, and hence $\|x\|_{\mathcal{P C}} \leq v(t) \leq \xi(t) \leq \widetilde{K}$, where $\widetilde{K}$ depends only on $M, \delta, b$ and on the functions $m(\cdot)$, $p(\cdot, \cdot), \Theta(\cdot)$ and $\Theta_{0}(\cdot)$. Then there exists $r^{*}$ such that $\|x\|_{\mathcal{P C}} \neq r^{*}$. Set

$$
V=\left\{x \in \mathcal{Y}:\|x\|_{\mathcal{P C}}<r^{*}\right\} .
$$

From the choice of $V$, there is no $x \in \partial V$ such that $x \in \lambda \Phi_{n} x$ for $\lambda \in(0,1)$. 
Step 2. $\Phi_{n}$ has a closed graph.

Let $x^{(j)} \rightarrow x^{*}, \rho_{n}^{(j)} \in \Phi_{n} x^{(j)}, x^{(j)} \in \bar{V}$ and $\rho_{n}^{(j)} \rightarrow \rho_{n}^{*}$. From axiom (A), it is easy to see that $\left.\overline{\left(x^{(j)}\right.}\right)_{s} \rightarrow \overline{x^{*}}{ }_{s}$ uniformly for $s \in(-\infty, b]$ as $n \rightarrow \infty$. We prove that $\rho_{n}^{*} \in \Phi_{n} \overline{x^{*}}$. Now $\rho_{n}^{(j)} \in$ $\Phi_{n} \overline{x^{(j)}}$ means that there exists $f^{(j)} \in S_{F, x^{(j)}}$ such that, for each $t \in\left[0, t_{1}\right]$

$$
\begin{aligned}
\rho_{n}^{(j)}(t)= & \left.\mathcal{R}_{\alpha}(t)\left[\varphi(0)-\mathcal{R}_{\alpha}\left(\sigma_{n}\right) G(0, \varphi)\right]+\mathcal{R}_{\alpha}\left(\sigma_{n}\right) G\left(t, \overline{x^{(j)}}\right)_{t}\right) \\
& +\int_{0}^{t} \mathcal{S}_{\alpha}(t-s) B B^{*} \mathcal{S}_{\alpha}^{*}\left(t_{1}-s\right) R\left(a, \Gamma_{0}^{t_{1}}\right)\left[x_{0}+\frac{x_{b}-x_{0}}{m+1}-\mathcal{R}_{\alpha}\left(t_{1}\right)[\varphi(0)\right. \\
& \left.-\mathcal{R}_{\alpha}\left(\sigma_{n}\right) G(0, \varphi)\right]-\mathcal{R}_{\alpha}\left(\sigma_{n}\right) G\left(t_{1},\left(\overline{x^{(j)}}\right)_{t_{1}}\right) \\
& \left.-\int_{0}^{t_{1}} \mathcal{S}_{\alpha}\left(t_{1}-\eta\right) f^{(j)}(\eta) d \eta\right] d s+\int_{0}^{t} \mathcal{S}_{\alpha}(t-s) f^{(j)}(s) d s, \quad t \in\left[0, t_{1}\right] .
\end{aligned}
$$

We must prove that there exists $f^{*} \in S_{F, \overline{x^{*}}}$ such that, for each $t \in\left[0, t_{1}\right]$,

$$
\begin{aligned}
\rho_{n}^{*}(t)= & \mathcal{R}_{\alpha}(t)\left[\varphi(0)-\mathcal{R}_{\alpha}\left(\sigma_{n}\right) G(0, \varphi)\right]+\mathcal{R}_{\alpha}\left(\sigma_{n}\right) G\left(t,\left(\overline{x^{*}}\right)_{t}\right) \\
& +\int_{0}^{t} \mathcal{S}_{\alpha}(t-s) B B^{*} \mathcal{S}_{\alpha}^{*}\left(t_{1}-s\right) R\left(a, \Gamma_{0}^{t_{1}}\right)\left[x_{0}+\frac{x_{b}-x_{0}}{m+1}\right. \\
& -\mathcal{R}_{\alpha}\left(t_{1}\right)\left[\varphi(0)-\mathcal{R}_{\alpha}\left(\sigma_{n}\right) G(0, \varphi)\right]-\mathcal{R}_{\alpha}\left(\sigma_{n}\right) G\left(t_{1},\left(\overline{x^{*}}\right)_{t_{1}}\right) \\
& \left.-\int_{0}^{t_{1}} \mathcal{S}_{\alpha}\left(t_{1}-\eta\right) f^{(j)}(\eta) d \eta\right] d s+\int_{0}^{t} \mathcal{S}_{\alpha}(t-s) f^{*}(s) d s .
\end{aligned}
$$

Now, for every $t \in\left[0, t_{1}\right]$, we have

$$
\begin{aligned}
\|\left(\rho_{n}^{(j)}(t)-\mathcal{R}_{\alpha}(t)\left[\varphi(0)-\mathcal{R}_{\alpha}\left(\sigma_{n}\right) G(0, \varphi)\right]-\mathcal{R}_{\alpha}\left(\sigma_{n}\right) G\left(t, \overline{x^{(j)}}\right)_{t}\right) \\
\quad-\int_{0}^{t} \mathcal{S}_{\alpha}(t-s) B B^{*} \mathcal{S}_{\alpha}^{*}\left(t_{1}-s\right) R\left(a, \Gamma_{0}^{t_{1}}\right)\left[x_{0}+\frac{x_{b}-x_{0}}{m+1}-\mathcal{R}_{\alpha}\left(t_{1}\right)[\varphi(0)\right. \\
\left.\left.\left.\quad-\mathcal{R}_{\alpha}\left(\sigma_{n}\right) G(0, \varphi)\right]-\mathcal{R}_{\alpha}\left(\sigma_{n}\right) G\left(t_{1},\left(\overline{x^{(j)}}\right)_{t_{1}}\right)\right] d s\right) \\
\quad-\left(\rho_{n}^{*}(t)-\mathcal{R}_{\alpha}(t)\left[\varphi(0)-\mathcal{R}_{\alpha}\left(\sigma_{n}\right) G(0, \varphi)\right]-\mathcal{R}_{\alpha}\left(\sigma_{n}\right) G\left(t,\left(\overline{x^{*}}\right)_{t}\right)\right. \\
\quad-\int_{0}^{t} \mathcal{S}_{\alpha}(t-s) B B^{*} \mathcal{S}_{\alpha}^{*}\left(t_{1}-s\right) R\left(a, \Gamma_{0}^{t_{1}}\right)\left[x_{0}+\frac{x_{b}-x_{0}}{m+1}-\mathcal{R}_{\alpha}\left(t_{1}\right)[\varphi(0)\right. \\
\left.\left.\left.-\mathcal{R}_{\alpha}\left(\sigma_{n}\right) G(0, \varphi)\right]-\mathcal{R}_{\alpha}\left(\sigma_{n}\right) G\left(t_{1},\left(\overline{x^{*}}\right)_{t_{1}}\right)\right] d s\right) \|_{\mathcal{P C}} \rightarrow 0 \quad \text { as } j \rightarrow \infty .
\end{aligned}
$$

Consider the linear continuous operator $\Psi: L^{1}\left(\left[0, t_{1}\right], H\right) \rightarrow C\left(\left[0, t_{1}\right], H\right)$,

$$
\begin{aligned}
\Psi(f)(t)= & \int_{0}^{t} \mathcal{S}_{\alpha}(t-s) B B^{*} \mathcal{S}_{\alpha}^{*}\left(t_{1}-s\right) R\left(a, \Gamma_{0}^{t_{1}}\right)\left[\int_{0}^{t_{1}} \mathcal{S}_{\alpha}\left(t_{1}-\eta\right) f(\eta) d \eta\right] d s \\
& +\int_{0}^{t} \mathcal{S}_{\alpha}(t-s) f(s) d s .
\end{aligned}
$$

From Lemma 3.1, it follows that $\Psi \circ S_{F}$ is a closed graph operator. Also, from the definition of $\Psi$, we have, for every $t \in\left[0, t_{1}\right]$, 


$$
\begin{aligned}
\rho_{n}^{(j)}(t) & -\mathcal{R}_{\alpha}(t)\left[\varphi(0)-\mathcal{R}_{\alpha}\left(\sigma_{n}\right) G(0, \varphi)\right]+\mathcal{R}_{\alpha}\left(\sigma_{n}\right) G\left(t,\left(\overline{x^{(j)}}\right)_{t}\right) \\
& -\int_{0}^{t} \mathcal{S}_{\alpha}(t-s) B B^{*} \mathcal{S}_{\alpha}^{*}\left(t_{1}-s\right) R\left(a, \Gamma_{0}^{t_{1}}\right)\left[x_{0}+\frac{x_{b}-x_{0}}{m+1}\right. \\
& \left.-\mathcal{R}_{\alpha}\left(t_{1}\right)\left[\varphi(0)-\mathcal{R}_{\alpha}\left(\sigma_{n}\right) G(0, \varphi)\right]-\mathcal{R}_{\alpha}\left(\sigma_{n}\right) G\left(t_{1},\left(\overline{x^{(j)}}\right)_{t_{1}}\right)\right] d s \in \Psi\left(S_{F, \bar{x}, \overline{(j)}}\right) .
\end{aligned}
$$

Since $\overline{x^{(j)}} \rightarrow \overline{x^{*}}$, for some $f^{*} \in S_{F, \overline{x^{*}}}$ it follows that, for every $t \in\left[0, t_{1}\right]$, we have

$$
\begin{aligned}
\rho_{n}^{*}(t) & -\mathcal{R}_{\alpha}(t)\left[\varphi(0)-\mathcal{R}_{\alpha}\left(\sigma_{n}\right) G(0, \varphi)\right]+\mathcal{R}_{\alpha}\left(\sigma_{n}\right) G\left(t,\left(\overline{x^{*}}\right)_{t}\right) \\
& -\int_{0}^{t} \mathcal{S}_{\alpha}(t-s) B B^{*} \mathcal{S}_{\alpha}^{*}\left(t_{1}-s\right) R\left(a, \Gamma_{0}^{t_{1}}\right)\left[x_{0}+\frac{x_{1}-x_{0}}{m+1}\right. \\
& \left.-\mathcal{R}_{\alpha}(t)\left[\varphi(0)-\mathcal{R}_{\alpha}\left(\sigma_{n}\right) G(0, \varphi)\right]-\mathcal{R}_{\alpha}\left(\sigma_{n}\right) G\left(t_{1},\left(\overline{x^{*}}\right)_{t_{1}}\right)\right] d s \\
= & \int_{0}^{t} \mathcal{S}_{\alpha}(t-s) B B^{*} \mathcal{S}_{\alpha}^{*}\left(t_{1}-s\right) R\left(a, \Gamma_{0}^{t_{1}}\right)\left[\int_{0}^{t_{1}} \mathcal{S}_{\alpha}\left(t_{1}-\eta\right) f^{*}(\eta) d \eta\right] d s \\
& +\int_{0}^{t} \mathcal{S}_{\alpha}(t-s) f^{*}(s) d s .
\end{aligned}
$$

Similarly, for any $t \in\left(t_{k}, t_{k+1}\right], k=1, \ldots, m$, we have

$$
\begin{aligned}
\rho_{n}^{(j)}(t)= & \left.\mathcal{R}_{\alpha}\left(t-t_{k}\right)\left[\overline{x^{(j)}}\left(t_{k}^{-}\right)+\mathcal{R}_{\alpha}\left(\sigma_{n}\right) I_{k}\left(\left(\overline{x^{(j)}}\right)_{t_{k}}\right)-\mathcal{R}_{\alpha}\left(\sigma_{n}\right) G\left(t_{k}, \overline{x^{(j)}}\right)_{t_{k}^{+}}\right)\right] \\
& \left.-\mathcal{R}_{\alpha}\left(\sigma_{n}\right) G\left(t, \overline{x^{(j)}}\right)_{t}\right) \\
& +\int_{t_{k}}^{t} \mathcal{S}_{\alpha}(t-s) B B^{*} \mathcal{S}_{\alpha}^{*}\left(t_{k+1}-s\right) R\left(a, \Gamma_{t_{k}}^{t_{k+1}}\right)\left[x_{0}+\frac{(k+1)\left(x_{b}-x_{0}\right)}{m+1}\right. \\
& -\mathcal{R}_{\alpha}\left(t_{k+1}-t_{k}\right)\left[\overline{x^{(j)}}\left(t_{k}^{-}\right)+\mathcal{R}_{\alpha}\left(\sigma_{n}\right) I_{k}\left(\left(\overline{x^{(j)}}\right)_{t_{k}}\right)-\mathcal{R}_{\alpha}\left(\sigma_{n}\right) G\left(t_{k},\left(\overline{x^{(j)}}\right)_{t_{k}^{+}}\right)\right] \\
& \left.-\mathcal{R}_{\alpha}\left(\sigma_{n}\right) G\left(t_{k+1},\left(\overline{x^{(j)}}\right)_{t_{k+1}}\right)-\int_{t_{k}}^{t_{k+1}} \mathcal{S}_{\alpha}\left(t_{k+1}-\eta\right) f^{(j)}(\eta) d \eta\right] d s \\
& +\int_{t_{k}}^{t} \mathcal{S}_{\alpha}(t-s) f^{(j)}(s) d s, \quad t \in\left(t_{k}, t_{k+1}\right] .
\end{aligned}
$$

We must prove that there exists $f^{*} \in S_{F, \overline{x^{*}}}$ such that, for each $t \in\left(t_{k}, t_{k+1}\right]$,

$$
\begin{aligned}
\rho_{n}^{*}(t)= & \left.\mathcal{R}_{\alpha}\left(t-t_{k}\right)\left[\overline{x^{*}}\left(t_{k}^{-}\right)+\mathcal{R}_{\alpha}\left(\sigma_{n}\right) I_{k}\left(\left(\overline{x^{*}}\right)_{t_{k}}\right)-\mathcal{R}_{\alpha}\left(\sigma_{n}\right) G\left(t_{k}, \overline{x^{*}}\right)_{t_{k}^{+}}\right)\right] \\
& \left.-\mathcal{R}_{\alpha}\left(\sigma_{n}\right) G\left(t, \overline{x^{*}}\right)_{t}\right) \\
& +\int_{t_{k}}^{t} \mathcal{S}_{\alpha}(t-s) B B^{*} \mathcal{S}_{\alpha}^{*}\left(t_{k+1}-s\right) R\left(a, \Gamma_{t_{k}}^{t_{k+1}}\right)\left[x_{0}+\frac{(k+1)\left(x_{b}-x_{0}\right)}{m+1}\right. \\
& -\mathcal{R}_{\alpha}\left(t_{k+1}-t_{k}\right)\left[\overline{x^{*}}\left(t_{k}^{-}\right)+\mathcal{R}_{\alpha}\left(\sigma_{n}\right) I_{k}\left(\left(\overline{x^{*}}\right)_{t_{k}}\right)\right]-\mathcal{R}_{\alpha}\left(\sigma_{n}\right) G\left(t_{k},\left(\overline{x^{*}}\right)_{t_{k}^{+}}\right) \\
& \left.-\mathcal{R}_{\alpha}\left(\sigma_{n}\right) G\left(t_{k+1},\left(\overline{x^{*}}\right)_{t_{k+1}}\right)-\int_{t_{k}}^{t_{k+1}} \mathcal{S}_{\alpha}\left(t_{k+1}-\eta\right) f^{*}(\eta) d \eta\right] d s \\
& +\int_{t_{k}}^{t} \mathcal{S}_{\alpha}(t-s) f^{*}(s) d s, \quad t \in\left(t_{k}, t_{k+1}\right] .
\end{aligned}
$$


Now, for every $t \in\left(t_{k}, t_{k+1}\right], k=1, \ldots, m$, we have

$$
\begin{aligned}
\|\left(\rho_{n}^{(j)}(t)-\mathcal{R}_{\alpha}\left(t-t_{k}\right)\left[\overline{x^{(j)}}\left(t_{k}^{-}\right)+\mathcal{R}_{\alpha}\left(\sigma_{n}\right) I_{k}\left(\left(\overline{x^{(j)}}\right)_{t_{k}}\right)-\mathcal{R}_{\alpha}\left(\sigma_{n}\right) G\left(t_{k},\left(\overline{x^{(j)}}\right)_{t_{k}^{+}}\right)\right]\right. \\
\left.\quad-\mathcal{R}_{\alpha}\left(\sigma_{n}\right) G\left(t, \overline{x^{(j)}}\right)_{t}\right) \\
\quad-\int_{t_{k}}^{t} \mathcal{S}_{\alpha}(t-s) B B^{*} \mathcal{S}_{\alpha}^{*}\left(t_{k+1}-s\right) R\left(a, \Gamma_{t_{k}}^{t_{k+1}}\right)\left[x_{0}+\frac{(k+1)\left(x_{1}-x_{0}\right)}{m+1}\right. \\
\quad-\mathcal{R}_{\alpha}\left(t_{k+1}-t_{k}\right)\left[\overline{x^{(j)}}\left(t_{k}^{-}\right)+\mathcal{R}_{\alpha}\left(\sigma_{n}\right) I_{k}\left(\left(\overline{x^{(j)}}\right)_{t_{k}}\right)-\mathcal{R}_{\alpha}\left(\sigma_{n}\right) G\left(t_{k},\left(\overline{x^{(j)}}\right)_{t_{k}^{+}}\right)\right] \\
\left.\left.\quad-\mathcal{R}_{\alpha}\left(\sigma_{n}\right) G\left(t_{k+1},\left(\overline{x^{(j)}}\right)_{t_{k+1}}\right)\right] d s\right)-\left(\rho_{n}^{*}(t)-\mathcal{R}_{\alpha}\left(t-t_{k}\right)\left[\overline{x^{*}}\left(t_{k}^{-}\right)+I_{k}\left(\left(\overline{x^{*}}\right)_{t_{k}}\right)\right.\right. \\
\left.\quad-\mathcal{R}_{\alpha}\left(\sigma_{n}\right) G\left(t_{k},\left(\overline{x^{*}}\right)_{t_{k}^{+}}\right)\right]-\mathcal{R}_{\alpha}\left(\sigma_{n}\right) G\left(t,\left(\overline{x^{*}}\right)_{t}\right) \\
\quad-\int_{t_{k}}^{t} \mathcal{S}_{\alpha}(t-s) B B^{*} \mathcal{S}_{\alpha}^{*}\left(t_{k+1}-s\right) R\left(a, \Gamma_{t_{k}}^{t_{k+1}}\right)\left[x_{0}+\frac{(k+1)\left(x_{1}-x_{0}\right)}{m+1}\right. \\
-\mathcal{R}_{\alpha}\left(t_{k+1}-t_{k}\right)\left[\overline{x^{*}}\left(t_{k}^{-}\right)+\mathcal{R}_{\alpha}\left(\sigma_{n}\right) I_{k}\left(\left(\overline{x^{*}}\right)_{t_{k}}\right)-\mathcal{R}_{\alpha}\left(\sigma_{n}\right) G\left(t_{k},\left(\overline{x^{*}}\right)_{t_{k}^{+}}\right)\right] \\
\left.\left.-\mathcal{R}_{\alpha}\left(\sigma_{n}\right) G\left(t_{k+1},\left(\overline{x^{*}}\right)_{t_{k+1}}\right)\right] d s\right) \|_{\mathcal{P C}} \rightarrow 0 \text { as } j \rightarrow \infty .
\end{aligned}
$$

Consider the linear continuous operator $\Psi: L^{1}\left(\left(t_{k}, t_{k+1}\right], H\right) \rightarrow C\left(\left(t_{k}, t_{k+1}\right], H\right), k=1, \ldots, m$,

$$
\begin{aligned}
\Psi(f)(t)= & \int_{t_{k}}^{t} \mathcal{S}_{\alpha}(t-s) B B^{*} \mathcal{S}_{\alpha}^{*}\left(t_{k+1}-s\right) R\left(a, \Gamma_{t_{k}}^{t_{k+1}}\right)\left[\int_{t_{k}}^{t_{k+1}} \mathcal{S}_{\alpha}\left(t_{1}-\eta\right) f(\eta) d \eta\right] d s \\
& +\int_{t_{k}}^{t} \mathcal{S}_{\alpha}(t-s) f(s) d s .
\end{aligned}
$$

From Lemma 3.1, it follows that $\Psi \circ S_{F}$ is a closed graph operator. Also, from the definition of $\Psi$, we have, for every $t \in\left(t_{k}, t_{k+1}\right], k=1, \ldots, m$,

$$
\begin{aligned}
\rho_{n}^{(j)}(t) & -\mathcal{R}_{\alpha}\left(t-t_{k}\right)\left[\overline{x^{(j)}}\left(t_{k}^{-}\right)+\mathcal{R}_{\alpha}\left(\sigma_{n}\right) I_{k}\left(\left(\overline{x^{(j)}}\right)_{t_{k}}\right)-\mathcal{R}_{\alpha}\left(\sigma_{n}\right) G\left(t_{k},\left(\overline{x^{(j)}}\right)_{t_{k}^{+}}\right)\right] \\
& \left.-\mathcal{R}_{\alpha}\left(\sigma_{n}\right) G\left(t, \overline{x^{(j)}}\right)_{t}\right) \\
& -\int_{t_{k}}^{t} \mathcal{S}_{\alpha}(t-s) B B^{*} \mathcal{S}_{\alpha}^{*}\left(t_{k+1}-s\right) R\left(a, \Gamma_{t_{k}}^{t_{k+1}}\right)\left[x_{0}+\frac{(k+1)\left(x_{b}-x_{0}\right)}{m+1}\right. \\
& -\mathcal{R}_{\alpha}\left(t_{k+1}-t_{k}\right)\left[\overline{x^{(j)}}\left(t_{k}^{-}\right)+\mathcal{R}_{\alpha}\left(\sigma_{n}\right) I_{k}\left(\left(\overline{x^{(j)}}\right)_{t_{k}}\right)-\mathcal{R}_{\alpha}\left(\sigma_{n}\right) G\left(t_{k},\left(\overline{x^{(j)}}\right)_{t_{k}^{+}}\right)\right] \\
& \left.-\mathcal{R}_{\alpha}\left(\sigma_{n}\right) G\left(t_{k+1},\left(\overline{x^{(j)}}\right)_{t_{k+1}}\right)\right] d s \in \Psi\left(S_{\left.F, \overline{x^{(j)}}\right)}\right)
\end{aligned}
$$

Since $\overline{x^{(j)}} \rightarrow \overline{x^{*}}$, for some $f^{*} \in S_{F, \overline{x^{*}}}$ it follows that, for every $t \in\left(t_{k}, t_{k+1}\right]$, we have

$$
\begin{aligned}
\rho_{n}^{*}(t) & -\mathcal{R}_{\alpha}\left(t-t_{k}\right)\left[\overline{x^{*}}\left(t_{k}^{-}\right)+\mathcal{R}_{\alpha}\left(\sigma_{n}\right) I_{k}\left(\left(\overline{x^{*}}\right)_{t_{k}}\right)-\mathcal{R}_{\alpha}\left(\sigma_{n}\right) G\left(t_{k},\left(\overline{x^{*}}\right)_{t_{k}^{+}}\right)\right] \\
& -\mathcal{R}_{\alpha}\left(\sigma_{n}\right) G\left(t,\left(\overline{x^{*}}\right)_{t}\right) \\
& -\int_{t_{k}}^{t} \mathcal{S}_{\alpha}(t-s) B B^{*} \mathcal{S}_{\alpha}^{*}\left(t_{k+1}-s\right) R\left(a, \Gamma_{t_{k}}^{t_{k+1}}\right)\left[x_{0}+\frac{(k+1)\left(x_{b}-x_{0}\right)}{m+1}\right.
\end{aligned}
$$




$$
\begin{aligned}
& -\mathcal{R}_{\alpha}\left(t_{k+1}-t_{k}\right)\left[\overline{x^{*}}\left(t_{k}^{-}\right)+\mathcal{R}_{\alpha}\left(\sigma_{n}\right) I_{k}\left(\left(\overline{x^{*}}\right)_{t_{k}}\right)-\mathcal{R}_{\alpha}\left(\sigma_{n}\right) G\left(t_{k},\left(\overline{x^{*}}\right)_{t_{k}^{+}}\right)\right] \\
& \left.-\mathcal{R}_{\alpha}\left(\sigma_{n}\right) G\left(t_{k+1},\left(\overline{x^{*}}\right)_{t_{k+1}}\right)\right] d s \\
= & \int_{t_{k}}^{t} \mathcal{S}_{\alpha}(t-s) B B^{*} \mathcal{S}_{\alpha}^{*}\left(t_{k+1}-s\right) R\left(a, \Gamma_{t_{k}}^{t_{k+1}}\right)\left[\int_{t_{k}}^{t_{k+1}} \mathcal{S}_{\alpha}\left(t_{k+1}-\eta\right) f^{*}(\eta) d \eta\right] d s \\
& +\int_{t_{k}}^{t} \mathcal{S}_{\alpha}(t-s) f^{*}(s) d s .
\end{aligned}
$$

Therefore, $\Phi_{n}$ has a closed graph.

Step 3. We show that the operator $\Phi_{n}$ is condensing.

For this purpose, we decompose $\Phi_{n}$ as $\Lambda_{n}+\Gamma_{n}$, where the map $\Lambda_{n}: \bar{V} \rightarrow \mathcal{Y}$ is defined by $\Lambda_{n} x$, the set $\varpi_{n} \in \mathcal{Y}$ is such that

$$
\varpi_{n}(t)= \begin{cases}0, & t \in(-\infty, 0], \\ -\mathcal{R}_{\alpha}(t) \mathcal{R}_{\alpha}\left(\sigma_{n}\right) G(0, \varphi)+\mathcal{R}_{\alpha}\left(\sigma_{n}\right) G\left(t, \bar{x}_{t}\right), & t \in\left[0, t_{1}\right], \\ -\mathcal{R}_{\alpha}\left(t-t_{1}\right) \mathcal{R}_{\alpha}\left(\sigma_{n}\right) G\left(t_{1}, \bar{x}_{t_{1}^{+}}\right)+\mathcal{R}_{\alpha}\left(\sigma_{n}\right) G\left(t, \bar{x}_{t}\right), & t \in\left(t_{1}, t_{2}\right], \\ \vdots & \\ -\mathcal{R}_{\alpha}\left(t-t_{m}\right) \mathcal{R}_{\alpha}\left(\sigma_{n}\right) G\left(t_{m}, \bar{x}_{t_{m}^{+}}\right)+\mathcal{R}_{\alpha}\left(\sigma_{n}\right) G\left(t, \bar{x}_{t}\right), & t \in\left(t_{m}, b\right],\end{cases}
$$

and the map $\Gamma_{n}: \bar{V} \rightarrow \mathcal{Y}$ is defined by $\Gamma_{n} x$, the set $\vartheta_{n} \in \mathcal{Y}$ is such that

$$
\vartheta_{n}(t)= \begin{cases}0, & t \in(-\infty, 0], \\ \mathcal{R}_{\alpha}(t) \varphi(0)+\int_{0}^{t} \mathcal{S}_{\alpha}(t-s) B u_{\bar{x}}^{a}(s) d s+\int_{0}^{t} \mathcal{S}_{\alpha}(t-s) f(s) d s, & t \in\left[0, t_{1}\right], \\ \mathcal{R}_{\alpha}\left(t-t_{1}\right)\left[\bar{x}\left(t_{1}^{-}\right)+\mathcal{R}_{\alpha}\left(\sigma_{n}\right) I_{1}\left(\bar{x}_{t_{1}}\right)\right] & \\ \quad \quad \int_{t_{1}}^{t} \mathcal{S}_{\alpha}(t-s) B u_{\bar{x}}^{a}(s) d s+\int_{t_{1}}^{t} \mathcal{S}_{\alpha}(t-s) f(s) d s, & t \in\left(t_{1}, t_{2}\right], \\ \vdots & \\ \mathcal{R}_{\alpha}\left(t-t_{m}\right)\left[\bar{x}\left(t_{m}^{-}\right)+\mathcal{R}_{\alpha}\left(\sigma_{n}\right) I_{m}\left(\bar{x}_{t_{m}}\right)\right] & \\ \quad+\int_{t_{m}}^{t} \mathcal{S}_{\alpha}(t-s) B u_{\bar{x}}^{a}(s) d s+\int_{t_{m}}^{t} \mathcal{S}_{\alpha}(t-s) f(s) d s, & t \in\left(t_{m}, b\right] .\end{cases}
$$

We first show that $\Lambda_{n}$ is a contraction while $\Gamma_{n}$ is a completely continuous operator.

Claim $1 . \Lambda_{n}$ is a contraction on $\bar{V}$.

Let $t \in\left[0, t_{1}\right]$ and $x^{*}, x^{* *} \in \mathcal{Y}$. From (H2), we have

$$
\begin{aligned}
\| & \left(\Lambda_{n} x^{*}\right)(t)-\left(\Lambda_{n} x^{* *}\right)(t) \| \\
& \leq\left\|\mathcal{R}_{\alpha}\left(\sigma_{n}\right)\left[G\left(t, \overline{x^{*}}\right)-G\left(t, \overline{x^{* *}}\right)\right]\right\| \\
& \leq L M e^{\delta \sigma_{n}}\left\|\overline{x^{*}}+\overline{x^{* *}}\right\|_{\mathcal{B}} \\
& \leq L M e^{\delta \sigma_{n}} K_{b} \sup _{s \in[0, b]}\left\|\overline{x^{*}}(s)-\overline{x^{* *}}(s)\right\| \\
& =L M e^{\delta \sigma_{n}} K_{b} \sup _{s \in[0, b]}\left\|x^{*}(s)-x^{* *}(s)\right\| \quad(\text { since } \bar{x}=x \text { on } J) \\
& =L M e^{\delta \sigma_{n}} K_{b}\left\|x^{*}-x^{* *}\right\|_{\mathcal{P C}} .
\end{aligned}
$$


Similarly, for any $t \in\left(t_{k}, t_{k+1}\right], k=1, \ldots, m$, we have

$$
\begin{aligned}
\|( & \left.\Lambda_{n} x^{*}\right)(t)-\left(\Lambda_{n} x^{* *}\right)(t) \| \\
\leq & \left\|\mathcal{R}_{\alpha}\left(t-t_{k}\right) \mathcal{R}_{\alpha}\left(\sigma_{n}\right)\left[-G\left(t_{k}, \bar{x}_{t_{k}^{+}}\right)+G\left(t_{k}, \overline{x^{* *}} t_{k}^{+}\right)\right]\right\| \\
& +\left\|\mathcal{R}_{\alpha}\left(\sigma_{n}\right)\left[G\left(t, \overline{x^{*}} t\right)-G\left(t, \overline{x^{* *}} t\right)\right]\right\| \\
\leq & M e^{\delta\left(t-t_{k}\right)} L M e^{\delta \sigma_{n}}\left\|\overline{x^{*}} t_{k}^{+}-\overline{x^{* *}} t_{k}^{+}\right\|_{\mathcal{B}}+L M e^{\delta \sigma_{n}}\left\|\overline{x^{*}}{ }_{t}-\overline{x^{* *}}\right\|_{\mathcal{B}} \\
\leq & \left(M_{*} N_{*}+1\right) L M e^{\delta \sigma_{n}} K_{b} \sup _{s \in[0, b]}\left\|\overline{x^{*}}(s)-\overline{x^{* *}}(s)\right\| \\
= & \left(M_{*} N_{*}+1\right) L M e^{\delta \sigma_{n}} K_{b} \sup _{s \in[0, b]}\left\|x^{*}(s)-x^{* *}(s)\right\| \quad(\text { since } \bar{x}=x \text { on } J) \\
= & \left(M_{*} N_{*}+1\right) L M e^{\delta \sigma_{n}} K_{b}\left\|x^{*}-x^{* *}\right\|_{\mathcal{P C}},
\end{aligned}
$$

where $M_{*}=M \max \left\{1, e^{\delta b}\right\}, N_{*}=\max \left\{1, e^{-\delta b}\right\}$. Thus, for all $t \in[0, b]$, we have

$$
\left\|\left(\Lambda_{n} x^{*}\right)(t)-\left(\Lambda_{n} x^{* *}\right)(t)\right\| \leq\left(M_{*} N_{*}+1\right) L M e^{\delta \sigma_{n}} K_{b}\left\|x^{*}-x^{* *}\right\|_{\mathcal{P C}} .
$$

Since $\lim _{n \rightarrow \infty} \sigma_{n}=0$, it follows that

$$
\left\|\left(\Lambda_{n} x^{*}\right)(t)-\left(\Lambda_{n} x^{* *}\right)(t)\right\| \leq L_{0}\left\|x^{*}-x^{* *}\right\|_{\mathcal{P C}}
$$

Taking the supremum over $t$,

$$
\left\|\Lambda_{n} x^{*}-\Lambda_{n} x^{* *}\right\|_{\mathcal{P C}} \leq L_{0}\left\|x^{*}-x^{* *}\right\|_{\mathcal{P C}}
$$

where $L_{0}=\left(M_{*} N_{*}+1\right) L M K_{b}$. By (3.2), we see that $L_{0}<1$. Hence, $\Lambda_{n}$ is a contraction on $\mathcal{Y}$.

Claim 2. $\Gamma_{n}$ is convex for each $x \in \bar{V}$.

In fact, if $\vartheta_{n}^{1}, \vartheta_{n}^{2}$ belong to $\Gamma_{n} x$, then there exist $f_{1}, f_{2} \in S_{F, \bar{x}}$ such that

$$
\begin{aligned}
\vartheta_{n}^{i}(t)= & \mathcal{R}_{\alpha}(t) \varphi(0)+\int_{0}^{t} S(t-s) B B^{*} \mathcal{S}_{\alpha}^{*}\left(t_{1}-s\right) R\left(a, \Gamma_{0}^{t_{1}}\right)\left[x_{0}+\frac{x_{b}-x_{0}}{m+1}\right. \\
& -\mathcal{R}_{\alpha}\left(t_{1}\right)\left[\varphi(0)-\mathcal{R}_{\alpha}\left(\sigma_{n}\right) G(0, \varphi)\right]-\mathcal{R}_{\alpha}\left(\sigma_{n}\right) G\left(t_{1}, \bar{x}_{t_{1}}\right) \\
& \left.-\int_{0}^{t_{1}} \mathcal{S}_{\alpha}\left(t_{1}-\eta\right) f_{i}(\eta) d \eta\right] d s+\int_{0}^{t} \mathcal{S}_{\alpha}(t-s) f_{i}(s) d s, \quad t \in\left[0, t_{1}\right], i=1,2 .
\end{aligned}
$$

Let $0 \leq \lambda \leq 1$. For each $t \in\left[0, t_{1}\right]$ we have

$$
\begin{aligned}
\left(\lambda \vartheta_{n}^{1}\right. & \left.+(1-\lambda) \vartheta_{n}^{2}\right)(t) \\
= & \mathcal{R}_{\alpha}(t) \varphi(0)+\int_{0}^{t} S(t-s) B B^{*} \mathcal{S}_{\alpha}^{*}\left(t_{1}-s\right) R\left(a, \Gamma_{0}^{t_{1}}\right)\left[x_{0}+\frac{x_{b}-x_{0}}{m+1}\right. \\
& -\mathcal{R}_{\alpha}(t)\left[\varphi(0)-\mathcal{R}_{\alpha}\left(\sigma_{n}\right) G(0, \varphi)\right]-\mathcal{R}_{\alpha}\left(\sigma_{n}\right) G\left(t_{1}, \bar{x}_{t_{1}}\right) \\
& \left.-\int_{0}^{t_{1}} \mathcal{S}_{\alpha}\left(t_{1}-\eta\right)\left[\lambda f_{1}(\eta)+(1-\lambda) f_{2}(\eta)\right] d \eta\right] d s \\
& +\int_{0}^{t} \mathcal{S}_{\alpha}(t-s)\left[\lambda f_{1}(s)+(1-\lambda) f_{2}(s)\right] d s
\end{aligned}
$$


Similarly, for any $t \in\left(t_{k}, t_{k+1}\right], k=1, \ldots, m$, we have

$$
\begin{aligned}
\vartheta_{n}^{i}(t)= & \mathcal{R}_{\alpha}\left(t-t_{k}\right)\left[\bar{x}\left(t_{k}^{-}\right)+\mathcal{R}_{\alpha}\left(\sigma_{n}\right) I_{k}\left(\bar{x}_{t_{k}}\right)\right] \\
& +\int_{t_{k}}^{t} S(t-s) B B^{*} \mathcal{S}_{\alpha}^{*}\left(t_{k+1}-s\right) R\left(a, \Gamma_{t_{k}}^{t_{k+1}}\right)\left[x_{0}+\frac{(k+1)\left(x_{b}-x_{0}\right)}{m+1}\right. \\
& -\mathcal{R}_{\alpha}\left(t_{k+1}-t_{k}\right)\left[\bar{x}\left(t_{k}^{-}\right)+\mathcal{R}_{\alpha}\left(\sigma_{n}\right) I_{k}\left(\bar{x}_{t_{k}}\right)-\mathcal{R}_{\alpha}\left(\sigma_{n}\right) G\left(t_{k}, \bar{x}_{t_{k}^{+}}\right)\right] \\
& \left.-\mathcal{R}_{\alpha}\left(\sigma_{n}\right) G\left(t_{k+1}, \bar{x}_{t_{k+1}}\right)-\int_{t_{k}}^{t_{k+1}} \mathcal{S}_{\alpha}\left(t_{k+1}-\eta\right) f_{i}(\eta) d \eta\right] d s \\
& +\int_{t_{k}}^{t} \mathcal{S}_{\alpha}(t-s) f_{i}(s) d s, \quad i=1,2
\end{aligned}
$$

Let $0 \leq \lambda \leq 1$. For each $t \in\left(t_{k}, t_{k+1}\right], k=1, \ldots, m$, we have

$$
\begin{aligned}
\left(\lambda \vartheta_{n}^{1}\right. & \left.+(1-\lambda) \vartheta_{n}^{2}\right)(t) \\
= & \mathcal{R}_{\alpha}\left(t-t_{k}\right)\left[\bar{x}\left(t_{k}^{-}\right)+\mathcal{R}_{\alpha}\left(\sigma_{n}\right) I_{k}\left(\bar{x}_{t_{k}}\right)\right] \\
& +\int_{t_{k}}^{t} S(t-s) B B^{*} \mathcal{S}_{\alpha}^{*}\left(t_{k+1}-s\right) R\left(a, \Gamma_{t_{k}}^{t_{k+1}}\right)\left[x_{0}+\frac{(k+1)\left(x_{1}-x_{0}\right)}{m+1}\right. \\
& -\mathcal{R}_{\alpha}\left(t_{k+1}-t_{k}\right)\left[\bar{x}\left(t_{k}^{-}\right)+\mathcal{R}_{\alpha}\left(\sigma_{n}\right) I_{k}\left(\bar{x}_{t_{k}}\right)-\mathcal{R}_{\alpha}\left(\sigma_{n}\right) G\left(t_{k}, \bar{x}_{t_{k}^{+}}\right)\right] \\
& \left.-\mathcal{R}_{\alpha}\left(\sigma_{n}\right) G\left(t_{k}, \bar{x}_{t_{k+1}}\right)-\int_{t_{k}}^{t_{k+1}} \mathcal{S}_{\alpha}\left(t_{k+1}-\eta\right)\left[\lambda f_{1}(\eta)+(1-\lambda) f_{2}(\eta)\right] d \eta\right] d s \\
& +\int_{t_{k}}^{t} \mathcal{S}_{\alpha}(t-s)\left[\lambda f_{1}(s)+(1-\lambda) f_{2}(s)\right] d s .
\end{aligned}
$$

Since $S_{F, \bar{x}}$ is convex (because $F$ has convex values) we have $\left(\lambda \vartheta_{n}^{1}+(1-\lambda) \vartheta_{n}^{2}\right) \in \Gamma_{n} x$.

Claim 3. $\Gamma_{n}(\bar{V})$ is completely continuous.

We begin by showing that $\Gamma_{n}(\bar{V})$ is equicontinuous. If $x \in \bar{V}$, it follows that

$$
\left\|\bar{x}_{s}\right\|_{\mathcal{B}} \leq M_{b}\|\varphi\|_{\mathcal{B}}+K_{b} r^{*}:=r^{\prime}
$$

Let $0<\tau_{1}<\tau_{2} \leq t_{1}$. Then we see for each $x \in \bar{V}$, and $\vartheta_{n} \in \Gamma_{n} x$, that there exists $f \in S_{F, \bar{x}}$ such that

$$
\vartheta_{n}(t)=\mathcal{R}_{\alpha}(t) \varphi(0)+\int_{0}^{t} \mathcal{S}_{\alpha}(t-s) B u_{\bar{x}}^{a}(s) d s+\int_{0}^{t} \mathcal{S}_{\alpha}(t-s) f(s) d s
$$

Then

$$
\begin{aligned}
\left\|\vartheta_{n}\left(\tau_{2}\right)-\vartheta_{n}\left(\tau_{1}\right)\right\| \\
\leq\left\|\left[\mathcal{R}_{\alpha}\left(\tau_{2}\right)-\mathcal{R}_{\alpha}\left(\tau_{1}\right)\right] \varphi(0)\right\|+\left\|\int_{0}^{\tau_{1}-\varepsilon}\left[\mathcal{S}_{\alpha}\left(\tau_{2}-s\right)-\mathcal{S}_{\alpha}\left(\tau_{1}-s\right)\right] B u_{\bar{x}}(s) d s\right\| \\
\quad+\left\|\int_{\tau_{1}-\varepsilon}^{\tau_{1}}\left[\mathcal{S}_{\alpha}\left(\tau_{2}-s\right)-\mathcal{S}_{\alpha}\left(\tau_{1}-s\right)\right] B u_{\bar{x}}(s) d s\right\|+\left\|\int_{\tau_{1}}^{\tau_{2}} \mathcal{S}_{\alpha}\left(\tau_{2}-s\right) B u_{\bar{x}}(s) d s\right\| \\
\quad+\left\|\int_{0}^{\tau_{1}-\varepsilon}\left[\mathcal{S}_{\alpha}\left(\tau_{2}-s\right)-\mathcal{S}_{\alpha}\left(\tau_{1}-s\right)\right] f(s) d s\right\|
\end{aligned}
$$




$$
\begin{aligned}
& +\left\|\int_{\tau_{1}-\varepsilon}^{\tau_{1}}\left[\mathcal{S}_{\alpha}\left(\tau_{2}-s\right)-\mathcal{S}_{\alpha}\left(\tau_{1}-s\right)\right] f(s) d s\right\|+\left\|\int_{\tau_{1}}^{\tau_{2}} \mathcal{S}_{\alpha}\left(\tau_{2}-s\right) f(s) d s\right\| \\
\leq & \left\|\left[\mathcal{R}_{\alpha}\left(\tau_{2}\right)-\mathcal{R}_{\alpha}\left(\tau_{1}\right)\right] \varphi(0)\right\|+\int_{0}^{\tau_{1}-\varepsilon}\left\|\mathcal{S}_{\alpha}\left(\tau_{2}-s\right)-\mathcal{S}_{\alpha}\left(\tau_{1}-s\right)\right\| N_{0} d s \\
& +2 M_{*} \int_{\tau_{1}-\varepsilon}^{\tau_{1}} e^{-\delta s} N_{0} d s+M e^{\delta \tau_{2}} \int_{\tau_{1}}^{\tau_{2}} e^{-\delta s} N_{0} d s \\
& +\Theta\left(r^{\prime}\right) \int_{0}^{\tau_{1}-\varepsilon}\left\|\mathcal{S}_{\alpha}\left(\tau_{2}-s\right)-\mathcal{S}_{\alpha}\left(\tau_{1}-s\right)\right\| m(s) d s \\
& +2 M_{*} \Theta\left(r^{\prime}\right) \int_{\tau_{1}-\varepsilon}^{\tau_{1}} e^{-\delta s} m(s) d s+M e^{\delta \tau_{2}} \Theta\left(r^{\prime}\right) \int_{\tau_{1}}^{\tau_{2}} e^{-\delta s} m(s) d s .
\end{aligned}
$$

Similarly, for any $\tau_{1}, \tau_{2} \in\left(t_{k}, t_{k+1}\right], \tau_{1}<\tau_{2}, k=1, \ldots, m$, we have

$$
\begin{aligned}
\vartheta_{n}(t)= & \mathcal{R}_{\alpha}\left(t-t_{k}\right)\left[\bar{x}\left(t_{k}^{-}\right)+\mathcal{R}_{\alpha}\left(\sigma_{n}\right) I_{k}\left(\bar{x}_{t_{k}}\right)\right] \\
& +\int_{t_{k}}^{t} \mathcal{S}_{\alpha}(t-s) B u_{\bar{x}}^{a}(s) d s+\int_{t_{k}}^{t} \mathcal{S}_{\alpha}(t-s) f(s) d s .
\end{aligned}
$$

Then

$$
\begin{aligned}
& \left\|\vartheta_{n}\left(\tau_{2}\right)-\vartheta_{n}\left(\tau_{1}\right)\right\| \\
& \leq\left\|\left[\mathcal{R}_{\alpha}\left(\tau_{2}\right)-\mathcal{R}_{\alpha}\left(\tau_{1}\right)\right]\left[\bar{x}\left(t_{k}^{-}\right)+\mathcal{R}_{\alpha}\left(\sigma_{n}\right) I_{k}\left(\bar{x}_{t_{k}}\right)\right]\right\| \\
& \quad+\left\|\int_{t_{k}}^{\tau_{1}-\varepsilon}\left[\mathcal{S}_{\alpha}\left(\tau_{2}-s\right)-\mathcal{S}_{\alpha}\left(\tau_{1}-s\right)\right] B u_{\bar{x}}^{a}(s) d s\right\| \\
& \quad+\left\|\int_{\tau_{1}-\varepsilon}^{\tau_{1}}\left[\mathcal{S}_{\alpha}\left(\tau_{2}-s\right)-\mathcal{S}_{\alpha}\left(\tau_{1}-s\right)\right] B u_{\bar{x}}^{a}(s) d s\right\|+\left\|\int_{\tau_{1}}^{\tau_{2}} \mathcal{S}_{\alpha}\left(\tau_{2}-s\right) B u_{\bar{x}}^{a}(s) d s\right\| \\
& \quad+\left\|\int_{t_{k}}^{\tau_{1}-\varepsilon}\left[\mathcal{S}_{\alpha}\left(\tau_{2}-s\right)-\mathcal{S}_{\alpha}\left(\tau_{1}-s\right)\right] f(s) d s\right\| \\
& \quad+\left\|\int_{\tau_{1}-\varepsilon}^{\tau_{1}}\left[\mathcal{S}_{\alpha}\left(\tau_{2}-s\right)-\mathcal{S}_{\alpha}\left(\tau_{1}-s\right)\right] f(s) d s\right\|+\left\|\int_{\tau_{1}}^{\tau_{2}} \mathcal{S}_{\alpha}\left(\tau_{2}-s\right) f(s) d s\right\| \\
& \leq\left\|\left[\mathcal{R}_{\alpha}\left(\tau_{2}\right)-\mathcal{R}_{\alpha}\left(\tau_{1}\right)\right]\left[\bar{x}\left(t_{k}^{-}\right)+\mathcal{R}_{\alpha}\left(\sigma_{n}\right) I_{k}\left(\bar{x}_{t_{k}}\right)\right]\right\| \\
& +\int_{t_{k}}^{\tau_{1}-\varepsilon}\left\|\mathcal{S}_{\alpha}\left(\tau_{2}-s\right)-\mathcal{S}_{\alpha}\left(\tau_{1}-s\right)\right\| N_{k} d s \\
& \quad+2 M_{*} \int_{\tau_{1}-\varepsilon}^{\tau_{1}} e^{-\delta s} N_{k} d s+M e^{\delta \tau_{2}} \int_{\tau_{1}}^{\tau_{2}} e^{-\delta s} N_{k} d s \\
& \quad+\Theta\left(r^{\prime}\right) \int_{t_{k}}^{\tau_{1}-\varepsilon}\left\|\mathcal{S}_{\alpha}\left(\tau_{2}-s\right)-\mathcal{S}_{\alpha}\left(\tau_{1}-s\right)\right\| m(s) d s \\
& +2 M_{*} \Theta\left(r^{\prime}\right) \int_{\tau_{1}-\varepsilon}^{\tau_{1}} e^{-\delta s} m(s) d s+M e^{\delta \tau_{2}} \Theta\left(r^{\prime}\right) \int_{\tau_{1}}^{\tau_{2}} e^{-\delta s} m(s) d s .
\end{aligned}
$$

The right-hand side of the above inequality is independent of $x \in \bar{V}$ and tends to zero as $\tau_{2} \rightarrow \tau_{1}$, with $\varepsilon$ is sufficiently small, since the compactness of $\mathcal{R}_{\alpha}(t), \mathcal{S}_{\alpha}(t)$ for $t>0$ implies imply the continuity in the uniform operator topology and the set $\left\{\mathcal{R}_{\alpha}\left(\sigma_{n}\right) I_{k}\left(\bar{x}_{t_{k}}\right)\right.$ : $x \in \bar{V}, k=1, \ldots, m\}$ is relatively compact in $H$. It remains to prove that the functions $\Gamma_{n} x$, 
$x \in \bar{V}$, are equicontinuous at $t=0$. Indeed, this is true since $\mathcal{R}_{\alpha}\left(\sigma_{n}\right)$ is a compact operator. Thus, the set $\left\{\Gamma_{n} x: x \in \bar{V}\right\}$ is equicontinuous.

Next, we prove that $\Gamma_{n}(\bar{V})(t)=\left\{\vartheta_{n}(t): \vartheta_{n}(t) \in \Gamma_{n}(\bar{V})\right\}$ is relatively compact for every $t \in[0, b]$.

To this end, we decompose $\Gamma_{n}$ by $\Gamma_{n}(\bar{V})=\Gamma_{n}^{1}(\bar{V})+\Gamma_{n}^{2}(\bar{V})$, where the map $\Gamma_{n}^{1}$ is defined by $\Gamma_{n}^{1} x, x \in \bar{V}$ the set $\vartheta_{n}^{1}$ such that

$$
\vartheta_{n}^{1}(t)= \begin{cases}\int_{0}^{t} S_{\alpha}(t-s) B u_{\bar{x}}^{a}(s) d s+\int_{0}^{t} S_{\alpha}(t-s) f(s) d s, & t \in\left[0, t_{1}\right], \\ \int_{t_{1}}^{t} S_{\alpha}(t-s) B u_{\bar{x}}^{a}(s) d s+\int_{t_{1}}^{t} S_{\alpha}(t-s) f(s) d s, & t \in\left(t_{1}, t_{2}\right], \\ \vdots & \\ \int_{t_{m}}^{t} S_{\alpha}(t-s) B u_{\bar{x}}^{a}(s) d s+\int_{t_{m}}^{t} S_{\alpha}(t-s) f(s) d s, & t \in\left(t_{m}, b\right],\end{cases}
$$

and the map $\Gamma_{n}^{2}$ is defined by $\Gamma_{n}^{2} x, x \in \bar{V}$ the set $\vartheta_{n}^{2}$ such that

$$
\vartheta_{n}^{2}(t)= \begin{cases}\mathcal{R}_{\alpha}(t) \varphi(0), & t \in\left[0, t_{1}\right], \\ \mathcal{R}_{\alpha}\left(t-t_{1}\right)\left[\bar{x}\left(t_{1}^{-}\right)+\mathcal{R}_{\alpha}\left(\sigma_{n}\right) I_{1}\left(\bar{x}_{t_{1}}\right)\right], & t \in\left(t_{1}, t_{2}\right], \\ \vdots & \\ \mathcal{R}_{\alpha}\left(t-t_{m}\right)\left[\bar{x}\left(t_{m}^{-}\right)+\mathcal{R}_{\alpha}\left(\sigma_{n}\right) I_{m}\left(\bar{x}_{t_{m}}\right)\right], & t \in\left(t_{m}, b\right] .\end{cases}
$$

We now prove that $\Gamma_{n}^{1}(\bar{V})(t)=\left\{\vartheta_{n}^{1}(t): \vartheta_{n}^{1}(t) \in \Gamma_{n}^{1}(\bar{V})\right\}$ is relatively compact for every $t \in$ $[0, b]$. Let $0<t \leq s \leq t_{1}$ be fixed and let $\varepsilon$ be a real number satisfying $0<\varepsilon<t$. For $x \in \bar{V}$, we define

$$
\vartheta_{n}^{1, \varepsilon}(t)=\int_{0}^{t-\varepsilon} S_{\alpha}(t-s) B u_{\bar{x}}^{a}(s) d s+\int_{0}^{t-\varepsilon} \mathcal{S}_{\alpha}(t-s) f(s) d s,
$$

where $f \in S_{F, \bar{x}}$. Using the compactness of $\mathcal{S}_{\alpha}(t)$ for $t>0$, we deduce that the set $U_{\varepsilon}(t)=$ $\left\{\vartheta_{n}^{1, \varepsilon}(t): x \in \bar{V}\right\}$ is relatively compact in $H$ for every $\varepsilon, 0<\varepsilon<t$. Moreover, for every $x \in \bar{V}$ we have

$$
\begin{aligned}
\left\|\vartheta_{n}^{1}(t)-\vartheta_{n}^{1, \varepsilon}(t)\right\| & \leq\left\|\int_{t-\varepsilon}^{t} \mathcal{S}_{\alpha}(t-s) B u_{\bar{x}}^{a}(s) d s\right\|+\left\|\int_{t-\varepsilon}^{t} \mathcal{S}_{\alpha}(t-s) f(s) d s\right\| \\
& \leq M_{*} \int_{t-\varepsilon}^{t} e^{-\delta s} N_{0} d s+M_{*} \Theta\left(r^{\prime}\right) \int_{t-\varepsilon}^{t} e^{-\delta s} m(s) d s .
\end{aligned}
$$

Similarly, for any $t \in\left(t_{k}, t_{k+1}\right]$ with $k=1, \ldots, m$. Let $t_{k}<t \leq s \leq t_{k+1}$ be fixed and let $\varepsilon$ be a real number satisfying $0<\varepsilon<t$. For $x \in \bar{V}$, we define

$$
\vartheta_{n}^{1, \varepsilon}(t)=\int_{t_{k}}^{t-\varepsilon} \mathcal{S}_{\alpha}(t-s) B u_{\bar{x}}^{a}(s) d s+\int_{t_{k}}^{t-\varepsilon} \mathcal{S}_{\alpha}(t-s) f(s) d s,
$$

where $f \in S_{F, \bar{x}}$. Using the compactness of $\mathcal{S}_{\alpha}(t)$ for $t>0$, we deduce that the set $U_{\varepsilon}(t)=$ $\left\{\vartheta_{n}^{1, \varepsilon}(t): x \in \bar{V}\right\}$ is relatively compact in $H$ for every $\varepsilon, 0<\varepsilon<t$. Moreover, for every $x \in \bar{V}$ we have

$$
\begin{aligned}
\left\|\vartheta_{n}^{1}(t)-\vartheta_{n}^{1, \varepsilon}(t)\right\| & \leq\left\|\int_{t-\varepsilon}^{t} \mathcal{S}_{\alpha}(t-s) B u_{\bar{x}}(s) d s\right\|+\left\|\int_{t-\varepsilon}^{t} \mathcal{S}_{\alpha}(t-s) f(s) d s\right\| \\
& \leq M_{*} \int_{t-\varepsilon}^{t} e^{-\delta s} N_{k} d s+M_{*} \Theta\left(r^{\prime}\right) \int_{t-\varepsilon}^{t} e^{-\delta s} m(s) d s
\end{aligned}
$$


The right-hand side of the above inequality tends to zero as $\varepsilon \rightarrow 0$. Since there are relatively compact sets arbitrarily close to the set $U(t)=\left\{\vartheta_{n}^{1}(t): x \in \bar{V}\right\}$. Hence, the ArzeláAscoli theorem shows that $\Gamma_{n}^{1}$ is a compact multivalued map.

Next, we show that $\Gamma_{n}^{2}(\bar{V})(t)=\left\{\vartheta_{n}^{2}(t): \vartheta_{n}^{2}(t) \in \Gamma_{2}(\bar{V})\right\}$ is relatively compact for every $t \in[0, b]$. For all $t \in\left[0, t_{1}\right]$, since $\vartheta_{n}^{2}(t)=\mathcal{R}_{\alpha}(t) \varphi(0)$, by (H1), it follows that $\left\{\vartheta_{n}^{2}(t): t \in\right.$ $\left.\left[0, t_{1}\right], x \in \bar{V}\right\}$ is a compact subset of $H$. On the other hand, for $t \in\left(t_{k}, t_{k+1}\right], k=1, \ldots, m$, and $x \in \bar{V}$, there exists $r^{\prime \prime}>0$ such that

$$
\left[\widehat{\vartheta_{n}^{2}}\right]_{k}(t) \in \begin{cases}\mathcal{R}_{\alpha}\left(t-t_{k}\right)\left[\bar{x}\left(t_{k}^{-}\right)+\mathcal{R}_{\alpha}\left(\sigma_{n}\right) I_{k}\left(\bar{x}_{t_{k}}\right)\right], & t \in\left(t_{k}, t_{k+1}\right), x \in B_{r^{\prime \prime}}(0, \mathcal{Y}), \\ \mathcal{R}_{\alpha}\left(t_{k+1}-t_{k}\right)\left[\bar{x}\left(t_{k}^{-}\right)+\mathcal{R}_{\alpha}\left(\sigma_{n}\right) I_{k}\left(\bar{x}_{t_{k}}\right)\right], & t=t_{k+1}, x \in B_{r^{\prime \prime}}(0, \mathcal{Y}), \\ \bar{x}\left(t_{k}^{-}\right)+\mathcal{R}_{\alpha}\left(\sigma_{n}\right) I_{k}\left(\bar{x}_{t_{k}}\right), & t=t_{k}, x \in B_{r^{\prime \prime}}(0, \mathcal{Y}),\end{cases}
$$

where $B_{r^{\prime \prime}}(0, \mathcal{Y})$ is a closed ball of radius $r^{\prime \prime}$. From (3.7) we obtain $I_{k}\left(\bar{x}_{t_{k}}\right)$ bounded in $H$. By the compactness of $\left(\mathcal{R}_{\alpha}(t)\right)_{t>0}$, we see $\left\{\mathcal{R}_{\alpha}\left(\sigma_{n}\right) I_{k}\left(\bar{x}_{t_{k}}\right): x \in B_{r^{\prime \prime}}(0, \mathcal{Y}), k=1,2, \ldots, m\right\}$ are relatively compact in $H$. Also, it follows that $\left[\widehat{\vartheta}_{n}^{2}\right]_{k}(t)$ is relatively compact in $H$, for all $t \in$ $\left[t_{k}, t_{k+1}\right], k=1, \ldots, m$. By Lemma 2.7 , we infer that $\Gamma_{n}^{2}(\bar{V})$ is relatively compact. Moreover, using the continuity of the operator $\mathcal{R}_{\alpha}(t)$, for all $t \in[0, b]$, we conclude that operator $\Gamma_{n}^{2}$ is also a compact multivalued map.

As a consequence of the above Steps 1-3, we conclude that $\Phi_{n}=\Lambda_{n}+\Gamma_{n}$ is a condensing map. All of the conditions of Lemma 2.8 are satisfied, we deduce that $\Phi_{n}$ has a fixed point $x_{n} \in \mathcal{Y}$, which is in turn a mild solution of problem (3.3)-(3.5). Then we have

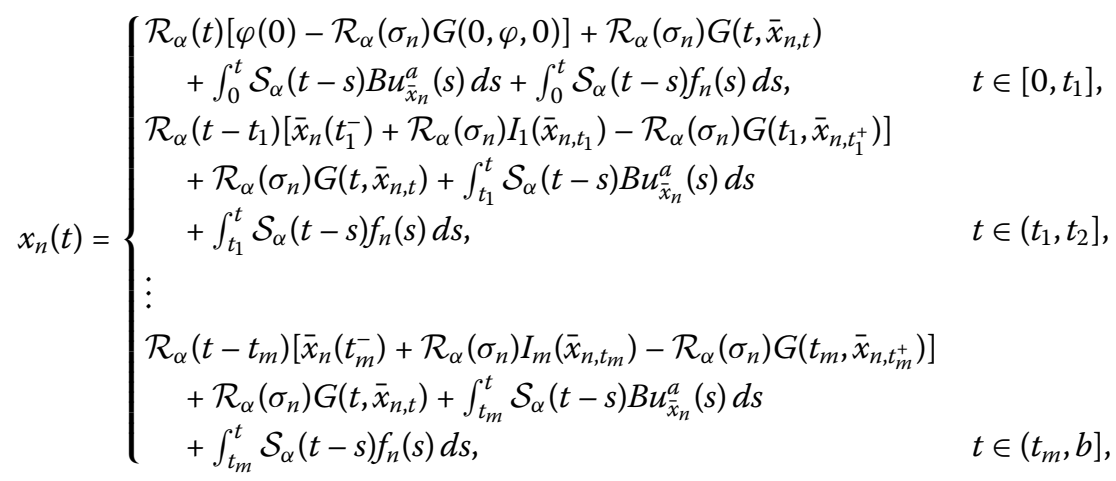

for $t \in[0, b]$, and some $f_{n} \in S_{F, \bar{x}_{n}}$.

Next we will show that the set $\left\{x_{n}: n \in \mathbb{N}\right\}$ is relatively compact in $\mathcal{Y}$. We consider the decomposition $x_{n}=x_{n}^{1}+x_{n}^{2}$ where

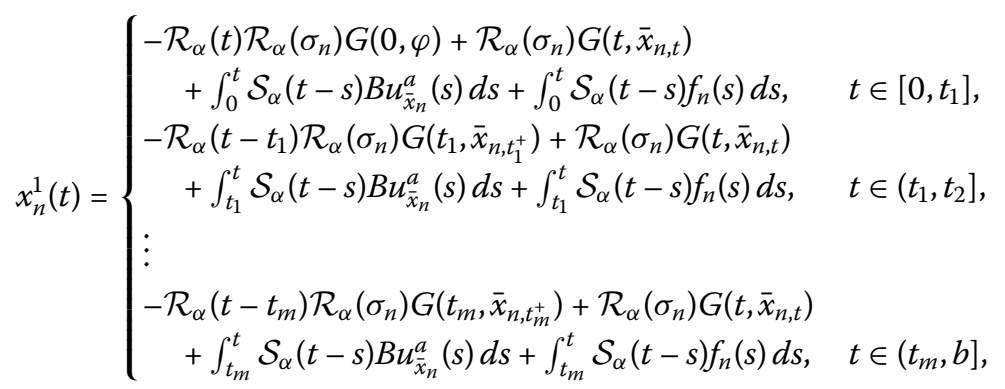


for some $f_{n} \in S_{F, \bar{x}_{n}}$, and

$$
x_{n}^{2}(t)= \begin{cases}\mathcal{R}_{\alpha}(t) \varphi(0), & t \in\left[0, t_{1}\right], \\ \mathcal{R}_{\alpha}\left(t-t_{1}\right)\left[\bar{x}_{n}\left(t_{1}^{-}\right)+\mathcal{R}_{\alpha}\left(\sigma_{n}\right) I_{1}\left(\bar{x}_{n, t_{1}}\right)\right], & t \in\left(t_{1}, t_{2}\right], \\ \vdots & \\ \mathcal{R}_{\alpha}\left(t-t_{m}\right)\left[\bar{x}_{n}\left(t_{m}^{-}\right)+\mathcal{R}_{\alpha}\left(\sigma_{n}\right) I_{m}\left(\bar{x}_{n, t_{m}}\right)\right], & t \in\left(t_{m}, b\right] .\end{cases}
$$

Step $4 .\left\{x_{n}^{1}(t): n \in \mathbb{N}\right\}$ is relatively compact in $\mathcal{Y}$.

Claim $1 .\left\{x_{n}^{1}: n \in \mathbb{N}\right\}$ is equicontinuous on $J$.

For $\varepsilon>0, x_{n} \in \bar{V}$, there exists a constant $\hat{\eta}>0$ such that, for all $t \in\left(0, t_{1}\right]$ and $\xi \in(0, \hat{\eta})$ with $t+\xi \leq t_{1}$, we have

$$
\begin{aligned}
\| x_{n}^{1}(t & +\xi)-x_{n}^{1}(t) \| \\
\leq & \left\|\left[\mathcal{R}_{\alpha}(t+\xi)-\mathcal{R}_{\alpha}(t)\right] \mathcal{R}_{\alpha}\left(\sigma_{n}\right) G(0, \varphi)\right\|+\left\|\mathcal{R}_{\alpha}\left(\sigma_{n}\right)\left[G\left(t+\xi, \bar{x}_{n, t+\xi},\right)-G\left(t, \bar{x}_{n, t}\right)\right]\right\| \\
& +\left\|\int_{t}^{t+\xi} \mathcal{S}_{\alpha}(t+\xi-s) B u_{\bar{x}_{n}}(s) d s\right\|+\left\|\int_{0}^{t}\left[\mathcal{S}_{\alpha}(t+\xi-s)-\mathcal{S}_{\alpha}(t-s)\right] B u_{\bar{x}_{n}}(s) d s\right\| \\
& +\left\|\int_{t}^{t+\xi} \mathcal{S}_{\alpha}(t+\xi-s) f_{n}(s) d s\right\|+\left\|\int_{0}^{t}\left[\mathcal{S}_{\alpha}(t+\xi-s)-\mathcal{S}_{\alpha}(t-s)\right] f_{n}(s) d s\right\| \\
\leq & \left\|\left[\mathcal{R}_{\alpha}(t+\xi)-\mathcal{R}_{\alpha}(t)\right] \mathcal{R}_{\alpha}\left(\sigma_{n}\right) G(0, \varphi, 0)\right\|+M e^{\delta \sigma_{n}} L\left[\xi+\left\|\bar{x}_{n, t+\xi}-\bar{x}_{n, t}\right\| \mathcal{B}\right] \\
& +M_{*} \int_{t}^{t+\xi} e^{-\delta s} N_{0} d s+\int_{0}^{t}\left\|\mathcal{S}_{\alpha}(t+\xi-s)-\mathcal{S}_{\alpha}(t-s)\right\| N_{0} d s \\
& +M_{*} \Theta\left(r^{\prime}\right) \int_{t}^{t+\xi} e^{-\sigma s} m(s) d s+\Theta\left(r^{\prime}\right) \int_{0}^{t}\left\|\mathcal{S}_{\alpha}(t+\xi-s)-\mathcal{S}_{\alpha}(t-s)\right\| m(s) d s .
\end{aligned}
$$

Similarly, for any $t \in\left(t_{k}, t_{k+1}\right], k=1, \ldots, m$, we have

$$
\begin{aligned}
\left\|x_{n}^{1}(t+\xi)-x_{n}^{1}(t)\right\| \\
\leq\left\|\left[\mathcal{R}_{\alpha}(t+\xi)-\mathcal{R}_{\alpha}(t)\right] \mathcal{R}_{\alpha}\left(\delta_{n}\right) G\left(t_{k}, \bar{x}_{n, t_{k}}\right)\right\|+M e^{\delta \sigma_{n}} L\left[\xi+\left\|\bar{x}_{n, t+\xi}-\bar{x}_{n, t}\right\|_{\mathcal{B}}\right] \\
\quad+M_{*} \int_{t}^{t+\xi} e^{-\sigma s} N_{k} d s+\int_{t_{k}}^{t}\left\|\mathcal{S}_{\alpha}(t+\xi-s)-\mathcal{S}_{\alpha}(t-s)\right\| N_{k} d s \\
\quad+M_{*} \Theta\left(r^{\prime}\right) \int_{t}^{t+\xi} e^{-\delta s} m(s) d s+\Theta\left(r^{\prime}\right) \int_{t_{k}}^{t}\left\|\mathcal{S}_{\alpha}(t+\xi-s)-\mathcal{S}_{\alpha}(t-s)\right\| m(s) d s .
\end{aligned}
$$

Then, for all $t \in(0, b]$, using the compact operator property, we get

$$
\left\|\left[\mathcal{R}_{\alpha}(t+\xi)-\mathcal{R}_{\alpha}(t)\right] \mathcal{R}_{\alpha}\left(\delta_{n}\right) G(0, \varphi)\right\|<\frac{\varepsilon}{6}
$$

or

$$
\left\|\left[\mathcal{R}_{\alpha}(t+\xi)-\mathcal{R}_{\alpha}(t)\right] \mathcal{R}_{\alpha}\left(\delta_{n}\right) G\left(t_{k}, \bar{x}_{n, t_{k}^{+}}\right)\right\|<\frac{\varepsilon}{6},
$$

and

$$
M e^{\delta \sigma_{n}} L\left[\xi+\left\|\bar{x}_{n, t+\xi}-\bar{x}_{n, t}\right\|_{\mathcal{B}}\right]<\frac{\varepsilon}{6},
$$




$$
\begin{aligned}
& M_{*} \int_{t}^{t+\xi} e^{-\delta s} N d s<\frac{\varepsilon}{6}, \\
& \int_{0}^{t}\left\|\mathcal{S}_{\alpha}(t+\xi-s)-\mathcal{S}_{\alpha}(t-s)\right\| N d s<\frac{\varepsilon}{6}, \\
& M_{*} \Theta\left(r^{\prime}\right) \int_{t}^{t+\xi} e^{-\delta s} m(s) d s<\frac{\varepsilon}{6}, \\
& \Theta\left(r^{\prime}\right) \int_{0}^{t}\left\|\mathcal{S}_{\alpha}(t+\xi-s)-\mathcal{S}_{\alpha}(t-s)\right\| m(s) d s<\frac{\varepsilon}{6},
\end{aligned}
$$

where $N=\max _{0 \leq k \leq m}\left\{N_{k}\right\}$. By (3.11)-(3.17) one has

$$
\left\|x_{n}^{1}(t+\xi)-x_{n}^{1}(t)\right\|<\varepsilon
$$

Therefore, $\left\{x_{n}^{1}(t): n \in \mathbb{N}\right\}$ is equicontinuous for $t \in(0, b]$. Clearly $\left\{x_{n}^{1}(0): n \in \mathbb{N}\right\}$ is equicontinuous.

Claim 2. $\left\{x_{n}^{1}(t): n \in \mathbb{N}\right\}$ is relatively compact in $H$.

Let $t \in\left(0, t_{1}\right], \varepsilon>0, x_{n} \in \bar{V}$, there exists $\xi \in(0, t)$ such that

$$
\begin{aligned}
\left\|x_{n}^{1}(t)-x_{n}^{\xi}(t)\right\| & \leq \int_{t-\xi}^{t}\left\|\mathcal{S}(t-s) B u_{\bar{x}_{n}}^{a}(s)\right\| d s+\int_{t-\xi}^{t}\left\|\mathcal{S}(t-s) f_{n}(s)\right\| d s \\
& \leq M_{*} \int_{t-\xi}^{t} e^{-\delta s} N_{0} d s+M_{*} \Theta\left(r^{\prime}\right) \int_{t-\xi}^{t} e^{-\delta s} m(s) d s<\varepsilon,
\end{aligned}
$$

where $x_{n}^{\xi}(t)=-\mathcal{R}_{\alpha}(t) \mathcal{R}_{\alpha}\left(\sigma_{n}\right) G(0, \varphi)+\mathcal{R}_{\alpha}\left(\sigma_{n}\right) G\left(t, \bar{x}_{n, t}\right)+\int_{0}^{t-\xi} \mathcal{S}_{\alpha}(t-s) f_{n}(s) d s$ for some $f_{n} \in$ $S_{F, \bar{x}_{n}}$.

Similarly, for any $t \in\left(t_{k}, t_{k+1}\right]$ with $k=1, \ldots, m . \varepsilon>0, x_{n} \in \bar{V}$, there exists $\xi \in(0, t)$ such that

$$
\begin{aligned}
\left\|x_{n}^{1}(t)-x_{n}^{\xi}(t)\right\| & \leq \int_{t-\xi}^{t}\left\|\mathcal{S}(t-s) B u_{\bar{x}_{n}}^{a}(s)\right\| d s+\int_{t-\xi}^{t}\left\|\mathcal{S}(t-s) f_{n}(s)\right\| d s \\
& \leq M_{*} \int_{t-\xi}^{t} e^{-\delta s} N_{k} d s+M_{*} \Theta\left(r^{\prime}\right) \int_{t-\xi}^{t} e^{-\delta s} m(s) d s<\varepsilon,
\end{aligned}
$$

where $x_{n}^{\xi}(t)=-\mathcal{R}_{\alpha}\left(t-t_{k}\right) \mathcal{R}_{\alpha}\left(\sigma_{n}\right) G\left(t_{k}, \bar{x}_{n, t_{k}^{+}}\right)+\mathcal{R}_{\alpha}\left(\sigma_{n}\right) G\left(t, \bar{x}_{n, t}\right)+\int_{t_{k}}^{t-\xi} \mathcal{S}_{\alpha}(t-s) f_{n}(s) d s$ for some $f_{n} \in S_{F, \bar{x}_{n}}$. From (H5), we obtain $G\left(t_{k}, \bar{x}_{n, t_{k}^{+}}\right), G\left(t, \bar{x}_{n, t}\right)$ are bounded in $H$. By the compactness of $\mathcal{R}_{\alpha}(t), \mathcal{S}_{\alpha}(t)$ for $t>0$, we see that the set $\left\{x_{n}^{\xi}(t): n \in \mathbb{N}\right\}$ is relatively compact in $H$. Combining the above inequality, one has $\left\{x_{n}^{1}(t): n \in \mathbb{N}\right\}$ is relatively compact in $H$.

Step $5 .\left\{x_{n}^{2}(t): n \in \mathbb{N}\right\}$ is relatively compact in $\mathcal{Y}$.

Claim $1 .\left\{x_{n}^{2}: n \in \mathbb{N}\right\}$ is equicontinuous on $J$.

For any $\varepsilon>0$ and $0<t<t_{1}$. Since $\mathcal{R}_{\alpha}\left(\sigma_{n}\right)$ is a compact operator, we find that the set $W_{1}=\left\{\mathcal{R}_{\alpha}\left(\sigma_{n}\right) G(0, \varphi)\right\}$ is relatively compact in $H$. From the strong continuity of $\left(\mathcal{R}_{\alpha}(t)\right)_{t \geq 0}$, for $\varepsilon>0$, we can choose $0<\hat{\eta}<b-t$ such that

$$
\left\|\left(\mathcal{R}_{\alpha}(t+\xi)-\mathcal{R}_{\alpha}(t)\right) v\right\|<\varepsilon, \quad v \in W_{1}
$$

when $|\xi|<\hat{\eta}$. For each $x_{n} \in \bar{V}, t \in\left(0, t_{1}\right)$, such that

$$
\left\|x_{n}^{2}(t+\xi)-x_{n}^{2}(t)\right\| \leq\left\|\left[\mathcal{R}_{\alpha}(t+\xi)-\mathcal{R}_{\alpha}(t)\right] \mathcal{R}_{\alpha}\left(\delta_{n}\right) G(0, \varphi)\right\|<\varepsilon
$$


Similarly, for any $t \in\left(t_{k}, t_{k+1}\right], k=1, \ldots, m$, and $\varepsilon>0$. Since $\mathcal{R}_{\alpha}\left(\sigma_{n}\right)$ is a compact operator, we find that the set $W_{2}=\left\{\mathcal{R}_{\alpha}\left(\sigma_{n}\right) I_{k}\left(\bar{x}_{n, t_{k}}\right): x_{n} \in \bar{V}\right\}$ is relatively compact in $H$. From the strong continuity of $\left(\mathcal{R}_{\alpha}(t)\right)_{t \geq 0}$, for $\varepsilon>0$, we can choose $0<\hat{\eta}<b-t$ such that

$$
\left\|\left(\mathcal{R}_{\alpha}(t+\xi)-\mathcal{R}_{\alpha}(t)\right) v\right\|<\frac{\varepsilon}{2(r+1)}, \quad v \in W_{2},
$$

when $|\xi|<\hat{\eta}$. For each $x_{n} \in \bar{V}, t \in\left(t_{k}, t_{k+1}\right], k=1, \ldots, m$, such that

$$
\begin{aligned}
\left\|x_{n}^{2}(t+\xi)-x_{n}^{2}(t)\right\| \leq & \left\|\mathcal{R}_{\alpha}\left(t+\xi-t_{k}\right)-\mathcal{R}_{\alpha}\left(t-t_{k}\right)\right\| r^{\prime} \\
& +\left\|\left[\mathcal{R}_{\alpha}\left(t+\xi-t_{k}\right)-\mathcal{R}_{\alpha}\left(t-t_{k}\right)\right] \mathcal{S}_{\alpha}\left(\sigma_{n}\right) I_{k}\left(\bar{x}_{n, t_{k}}\right)\right\|<\varepsilon .
\end{aligned}
$$

As $\xi \rightarrow 0$ and $\varepsilon$ sufficiently small, the right-hand side of the above inequality tends to zero independently of $x_{n}$, so $\left[\widehat{x_{n}^{2}}\right]_{k}, k=1,2, \ldots, m$, are equicontinuous.

Claim 2. $\left\{x_{n}^{2}(t): n \in \mathbb{N}\right\}$ is relatively compact in $H$.

Let $t \in\left(0, t_{1}\right], x_{n} \in \bar{V}$, by $(\mathrm{H} 1)$, it follows that $\left\{x_{n}^{2}(t): t \in\left[0, t_{1}\right], x_{n} \in \bar{V}\right\}$ is a compact subset of $X$. Using similar arguments to Step 2, for $t \in\left(t_{k}, t_{k+1}\right], k=1, \ldots, m$, and $x_{n} \in \bar{V}$, such that

$$
\left[\widehat{x_{n}^{2}}\right]_{k}(t) \in \begin{cases}\mathcal{R}_{\alpha}\left(t-t_{k}\right)\left[\bar{x}_{n}\left(t_{k}^{-}\right)+\mathcal{R}_{\alpha}\left(\sigma_{n}\right) I_{k}\left(\bar{x}_{n, t_{k}}\right)\right], & t \in\left(t_{k}, t_{k+1}\right), x_{n} \in B_{r^{\prime \prime}}(0, \mathcal{Y}), \\ \mathcal{R}_{\alpha}\left(t_{k+1}-t_{k}\right)\left[\bar{x}_{n}\left(t_{k}^{-}\right)+\mathcal{R}_{\alpha}\left(\sigma_{n}\right) I_{k}\left(\bar{x}_{n, t_{k}}\right)\right], & t=t_{k+1}, x_{n} \in B_{r^{\prime \prime}}(0, \mathcal{Y}), \\ \bar{x}_{n}\left(t_{k}^{-}\right)+\mathcal{R}_{\alpha}\left(\sigma_{n}\right) I_{k}\left(\bar{x}_{n, t_{k}}\right), & t=t_{k}, x_{n} \in B_{r^{\prime \prime}}(0, \mathcal{Y})\end{cases}
$$

where $B_{r^{\prime \prime}}(0, \mathcal{Y})$ is a closed ball of radius $r^{\prime \prime}$. One sees that $\left[\widehat{x_{n}^{2}}\right]_{k}(t), k=1,2, \ldots, m$, is relatively compact for every $t \in\left[t_{k}, t_{k+1}\right]$, and $\left\{x_{n}^{2}(t): n \in \mathbb{N}\right\}$ is relatively compact in $H$.

Thus, we obtain the result that the set $\left\{x_{n}: n \in \mathbb{N}\right\}$ is relatively compact in $\mathcal{Y}$. We may suppose that

$$
x_{n} \rightarrow x_{*} \in \mathcal{Y} \quad \text { as } n \rightarrow \infty
$$

Obviously, $x_{*} \in \mathcal{Y}$, taking the limits in (3.10) one has

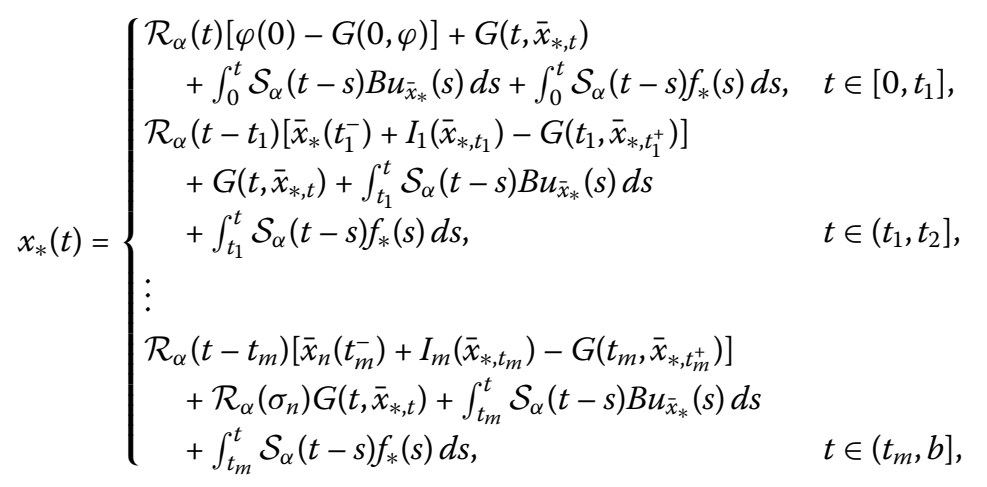

for $t \in[0, b]$, and some $f_{*} \in S_{F, \bar{x}_{*}}$. This implies that $x_{*}$ is a mild solution of problem (1.1)(1.3) and the proof of Theorem 3.1 is complete.

\section{Approximate controllability of fractional impulsive control systems}

In this section, we present our main result on the approximate controllability of system (1.1)-(1.3). To do this, we also need the following assumption. 
(B1) There exists a constant $\widetilde{C}>0$ such that

$$
\|F(t, \psi, y)\|=\sup \{\|f\|: f \in F(t, \psi, y)\} \leq \widetilde{C}, \quad(t, \psi, y) \in J \times \mathcal{B} \times H
$$

Theorem 4.1 Assume that assumptions of Theorem 3.1 hold and, in addition, assumption (B1) is satisfied and the linear system corresponding to system (1.1)-(1.3) is approximately controllable on J. Then system (1.1)-(1.3) is approximately controllable on J.

Proof Let $x^{a}(\cdot)$ be a fixed point of $\Phi$ in $\mathcal{Y}$. By Theorem 3.1, any fixed point of $\Phi$ is a mild solution of system (1.1)-(1.3). This means that there is $x^{a} \in \Phi\left(x^{a}\right)$, that is, there is $f \in S_{F, \bar{x}^{a}}$ such that

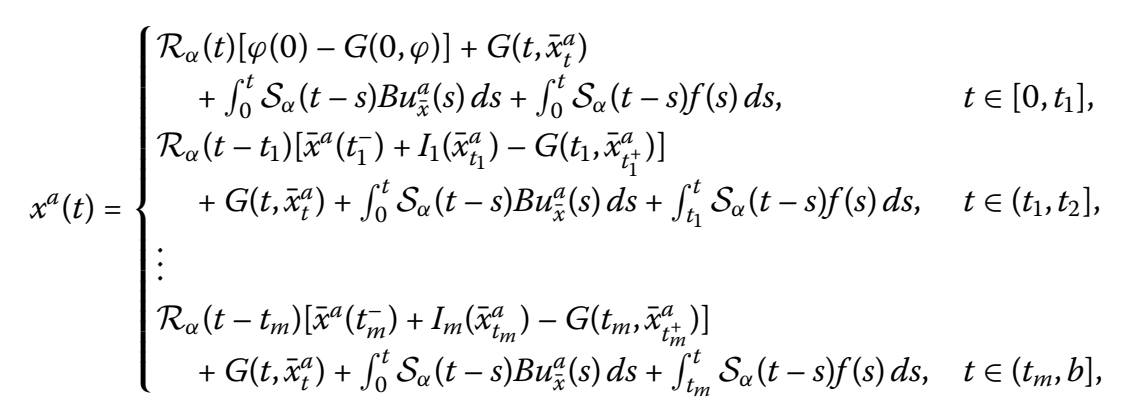

where

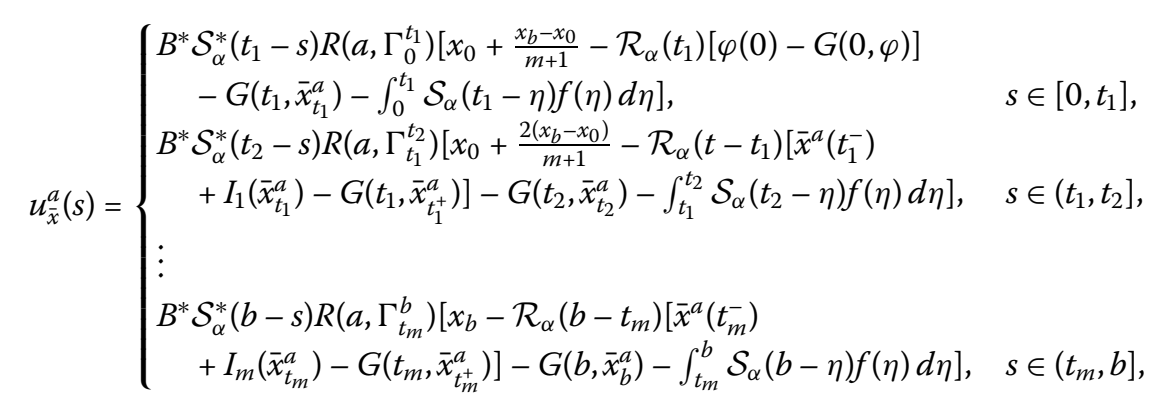

and it satisfies

$$
\left\{\begin{aligned}
x^{a}\left(t_{1}\right)= & x_{0}+\frac{x_{b}-x_{0}}{m+1}+a R\left(a, \Gamma_{0}^{t_{1}}\right)\left[x_{0}+\frac{x_{b}-x_{0}}{m+1}-\mathcal{R}_{\alpha}\left(t_{1}\right)[\varphi(0)-G(0, \varphi)]\right. \\
& \left.-G\left(t_{1}, \bar{x}_{t_{1}}^{a}\right)-\int_{0}^{t_{1}} \mathcal{S}_{\alpha}\left(t_{1}-\eta\right) f(\eta) d \eta\right] \\
x^{a}\left(t_{2}\right)= & x_{0}+\frac{2\left(x_{b}-x_{0}\right)}{m+1}+a R\left(a, \Gamma_{t_{1}}^{t_{2}}\right)\left[x_{0}+\frac{2\left(x_{b}-x_{0}\right)}{m+1}\right. \\
& -\mathcal{R}_{\alpha}\left(t_{2}-t_{1}\right)\left[\bar{x}^{a}\left(t_{1}^{-}\right)+I_{1}\left(\bar{x}_{t_{1}}^{a}\right)-G\left(t_{1}, \bar{x}_{t_{1}^{+}}^{a}\right)\right] \\
& \left.-G\left(t_{2}, \bar{x}_{t_{2}}^{a}\right)-\int_{t_{1}}^{t_{2}} \mathcal{S}_{\alpha}\left(t_{2}-\eta\right) f(\eta) d \eta\right] \\
\vdots & \\
x^{a}(b)= & x_{b}+a R\left(a, \Gamma_{t_{m}}^{b}\right)\left[x_{b}-\mathcal{R}_{\alpha}\left(b-t_{m}\right)\left[\bar{x}^{a}\left(t_{m}^{-}\right)+I_{m}\left(\bar{x}_{t_{m}}^{a}\right)\right.\right. \\
& \left.\left.-G\left(t_{m}, \bar{x}_{t_{m}^{+}}^{a}\right)\right]-G\left(b, \bar{x}_{b}^{a}\right)-\int_{t_{m}}^{b} \mathcal{S}_{\alpha}(b-\eta) f(\eta) d \eta\right]
\end{aligned}\right.
$$

By condition (B1), we see that

$$
\int_{0}^{b}\|f(s)\|^{2} d s \leq \widetilde{C}^{2} b
$$


Consequently, the sequence $\{f(\eta)\}$ is bounded in $L^{2}([0, b], H)$. Thus there are subsequences, still denoted by $\{f(\eta)\}$, that converge weakly to, say, $f^{* *}(\eta)$ in $L^{2}([0, b], H)$. The operator

$$
l(t) \rightarrow \int_{0}^{t} \mathcal{S}_{\alpha}(t-\eta) l(\eta) d \eta
$$

is also compact on $L^{2}([0, b], H)$, so one has

$$
\int_{0}^{t} \mathcal{S}_{\alpha}(t-\eta)\left[f(\eta)-f^{* *}(\eta)\right] d \eta \rightarrow 0 \quad \text { as } a \rightarrow 0^{+}
$$

for all $t \in[0, b]$.

Define

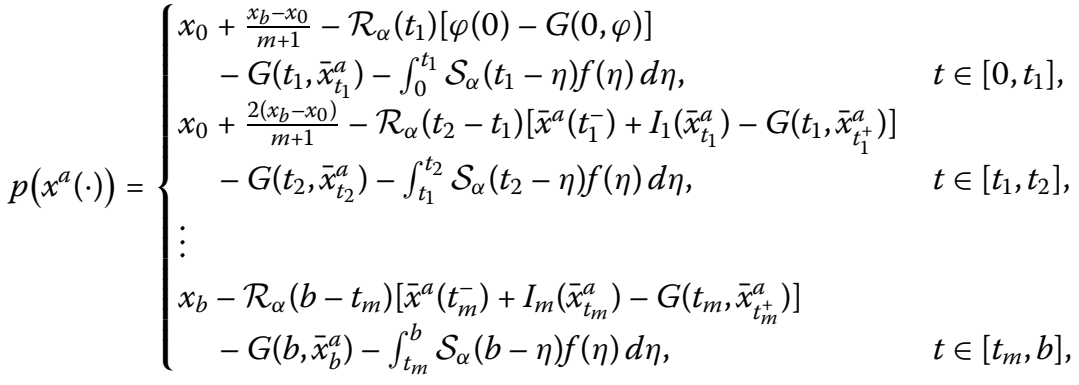

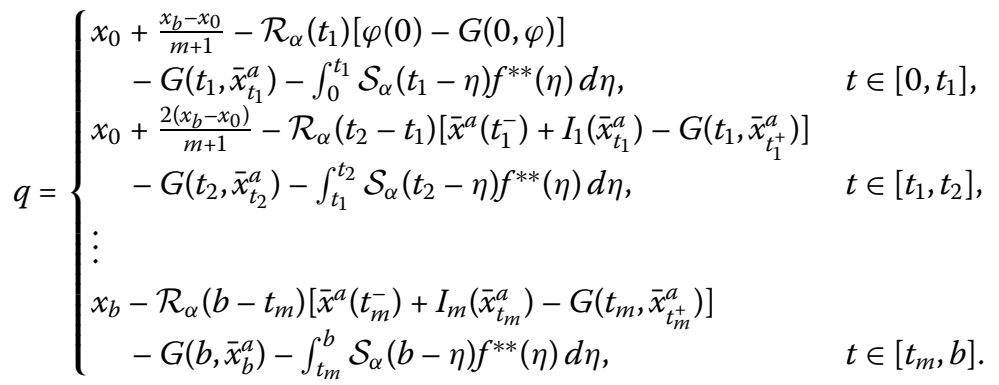

Hence, for all $t \in[0, b]$, we find that $\left\|p\left(x^{a}\right)-q\right\| \rightarrow 0$ as $a \rightarrow 0^{+}$. Moreover, from Lemma 2.6, we get, for $t \in\left[0, t_{1}\right]$,

$$
\begin{aligned}
\left\|x^{a}\left(t_{1}\right)-x_{t_{1}}\right\| & =\left\|a R\left(a, \Gamma_{0}^{t_{1}}\right)\left(x^{a}\right)\right\| \\
& \leq\left\|a R\left(a, \Gamma_{0}^{t_{1}}\right) q\right\|+\left\|a R\left(a, \Gamma_{0}^{t_{1}}\right)\left[p\left(x^{a}\right)-q\right]\right\| \\
& \leq\left\|a R\left(a, \Gamma_{0}^{t_{1}}\right) q\right\|+\left\|p\left(x^{a}\right)-q\right\| \rightarrow 0 \quad \text { as } a \rightarrow 0^{+} .
\end{aligned}
$$

Similarly, for any $t \in\left(t_{k}, t_{k+1}\right], k=1, \ldots, m$, we have

$$
\begin{aligned}
\left\|x^{a}\left(t_{k+1}\right)-x_{t_{k+1}}\right\| & =\left\|a R\left(a, \Gamma_{t_{k}}^{t_{k+1}}\right)\left(x^{a}\right)\right\| \\
& \leq\left\|a R\left(a, \Gamma_{t_{k}}^{t_{k+1}}\right) q\right\|+\left\|a R\left(a, \Gamma_{t_{k}}^{t_{k+1}}\right)\left[p\left(x^{a}\right)-q\right]\right\| \\
& \leq\left\|a R\left(a, \Gamma_{t_{k}}^{t_{k+1}}\right) q\right\|+\left\|p\left(x^{a}\right)-q\right\| \rightarrow 0 \quad \text { as } a \rightarrow 0^{+} .
\end{aligned}
$$

Thus, for all $t \in[0, b]$, we get $\left\|x^{a}(b)-x_{b}\right\| \rightarrow 0$ as $a \rightarrow 0^{+}$. This proves the approximate controllability of system (1.1)-(1.3). 


\section{Application}

Consider a fractional impulsive fractional partial neutral functional integrodifferential inclusions of the form

$$
\begin{aligned}
& \begin{aligned}
D_{t}^{\alpha} N\left(z_{t}\right)(x) \in & \frac{\partial^{2}}{\partial x^{2}} N\left(z_{t}\right)(x)+\int_{0}^{t}(t-s)^{\sigma} e^{-\mu(t-s)} \frac{\partial^{2}}{\partial x^{2}} N\left(z_{s}\right)(x) d s+\tilde{u}(t, x) \\
& \quad+\int_{-\infty}^{t} b_{2}(t, s-t, x, z(s, x)) d s \\
& -\int_{0}^{t} \int_{-\infty}^{s} b_{3}(t) b_{4}(s, \tau-s, x, z(\tau, x)) d \tau d s, \quad 0 \leq t \leq b, 0 \leq x \leq \pi,
\end{aligned} \\
& z(t, 0)=z(t, \pi)=0, \quad 0 \leq t \leq b, \\
& z_{t}(0, x)=0, \quad 0 \leq x \leq \pi \\
& z(\tau, x)=\varphi(\tau, x), \quad \tau \leq 0,0 \leq x \leq \pi, \\
& \Delta z\left(t_{k}, x\right)=\int_{-\infty}^{t_{k}} \eta_{k}\left(s-t_{k}\right) z(s, x) d s, \quad k=1,2, \ldots, m,
\end{aligned}
$$

where $D_{t}^{\alpha}$ is a Caputo fractional partial derivative of order $\alpha \in(1,2), \sigma$, and $\mu$ are positive numbers, and $\tilde{u}(\cdot)$ is a real function of bounded variation on $[0, b]$. In this system,

$$
N\left(z_{t}\right)(x)=z(t, x)-\int_{-\infty}^{t} b_{1}(s-t) z(s, x) d s
$$

Let $H=L^{2}([0, \pi])$ with the norm $\|\cdot\|$ and define the operators $A: D(A) \subseteq H \rightarrow H$ by $A \omega=\omega^{\prime \prime}$ with the domain

$$
D(A):=\left\{\omega \in H: \omega, \omega^{\prime} \text { are absolutely continuous, } \omega^{\prime \prime} \in H, \omega(0)=\omega(\pi)=0\right\} .
$$

Then

$$
A \omega=\sum_{n=1}^{\infty} n^{2}\left\langle\omega, \omega_{n}\right\rangle \omega_{n}, \quad \omega \in D(A),
$$

where $\omega_{n}(x)=\sqrt{\frac{2}{\pi}} \sin (n x), n=1,2, \ldots$ is the orthogonal set of eigenvectors of $A$. It is well known that $A$ generates a strongly continuous semigroup $T(t), t \geq 0$ which is compact, analytic and self-adjoint in $X$ and $A$ is sectorial of type and (P1) is satisfied. The operator $Q(t): D(A) \subseteq H \rightarrow H, t \geq 0, Q(t) x=t^{\sigma} e^{-\mu t} x^{\prime \prime}$ for $x \in D(A)$. Moreover, it is easy to see that conditions (P2) and (P3) in Section 2 are satisfied with $b(t)=t^{\sigma} e^{-\mu t}$ and $D=C_{0}^{\infty}([0, \pi])$, where $C_{0}^{\infty}([0, \pi])$ is the space of infinitely differentiable functions that vanish at $x=0$ and $x=\pi$.

Let $r \geq 0,1 \leq p<1$ and let $h:(-\infty,-r] \rightarrow \mathbb{R}$ be a nonnegative measurable function which satisfies the conditions (h-5), (h-6) in the terminology of Hino et al. [45]. Briefly, this means that $h$ is locally integrable and there is a nonnegative, locally bounded function $\gamma$ on $(-\infty, 0]$ such that $h(\xi+\tau) \leq \gamma(\xi) h(\tau)$ for all $\xi \leq 0$ and $\xi \in(-\infty,-r) \backslash N_{\xi}$, where $N_{\xi} \subseteq(-\infty,-r)$ is a set whose Lebesgue measure zero. We denote by $\mathcal{P} \mathcal{C}_{r} \times L^{p}(h, H)$ the set consisting of all classes of functions $\varphi:(-\infty, 0] \rightarrow H$ such that $\varphi_{[-r, 0]} \in \mathcal{P C}([-r, 0], H)$, 
$\varphi(\cdot)$ is Lebesgue measurable on $(-\infty,-r)$, and $h\|\varphi\|^{p}$ is Lebesgue integrable on $(-\infty,-r)$. The semi-norm is given by

$$
\|\varphi\|_{\mathcal{B}}=\sup _{-r \leq \tau \leq 0}\|\varphi(\tau)\|+\left(\int_{-\infty}^{-r} h(\tau)\|\varphi(\tau)\|^{p} d \tau\right)^{1 / p} .
$$

The space $\mathcal{B}=\mathcal{P C}_{r} \times L^{p}(h, H)$ satisfies axioms (A)-(C). Moreover, when $r=0$ and $p=2$, we can take $\tilde{H}=1, M(t)=\gamma(-t)^{1 / 2}$ and $K(t)=1+\left(\int_{-t}^{0} h(\tau) d \tau\right)^{1 / 2}$ for $t \geq 0$ (see [45], Theorem 1.3.8, for details).

Additionally, we will assume that

(i) The functions $b_{1}, b_{3}: \mathbb{R} \rightarrow \mathbb{R}$ are continuous, and $L_{G}=\left(\int_{-\infty}^{0} \frac{\left(b_{1}(s)\right)^{2}}{h(s)} d s\right)^{\frac{1}{2}}<\infty$.

(ii) The functions $b_{2}, b_{4}: \mathbb{R}^{4} \rightarrow \mathbb{R}$ are continuous and there exist continuous functions $a_{1}, a_{2}, a_{3}, a_{4}: \mathbb{R} \rightarrow \mathbb{R}$ such that

$$
\begin{array}{ll}
\left|b_{2}(t, s, x, y)\right| \leq a_{1}(t) a_{2}(s)|y|, & (t, s, x, y) \in \mathbb{R}^{4}, \\
\left|b_{4}(t, s, x, y)\right| \leq a_{3}(t) a_{4}(s)|y|, & (t, s, x, y) \in \mathbb{R}^{4},
\end{array}
$$

with $\widetilde{L}_{1}=\left(\int_{-\infty}^{0} \frac{\left(a_{2}(s)\right)^{2}}{h(s)} d s\right)^{\frac{1}{2}}<\infty, \widetilde{L}_{2}=\left(\int_{-\infty}^{0} \frac{\left(a_{4}(s)\right)^{2}}{h(s)} d s\right)^{\frac{1}{2}}<\infty$.

(iii) The functions $\eta_{k}: \mathbb{R} \rightarrow \mathbb{R}, k=1,2, \ldots, m$, are continuous, and

$$
L_{k}=\left(\int_{-\infty}^{0} \frac{\left(\eta_{k}(s)\right)^{2}}{h(s)} d s\right)^{\frac{1}{2}}<\infty \text { for every } k=1,2, \ldots, m
$$

Take $\varphi \in \mathcal{B}=\mathcal{P C}_{0} \times L^{2}(h, H)$ with $\varphi(s)(\psi)=\varphi(s, \psi)$. Let $N, G: \mathcal{B} \rightarrow H, F:[0, b] \times \mathcal{B} \times$ $H \rightarrow \mathcal{P}_{b d, c l, c v}(H)$ be the operators defined by

$$
\begin{aligned}
& N(\psi)(x)=\psi(0, x)+G(\psi)(x), \\
& G(\psi)(x)=\int_{-\infty}^{0} b_{1}(s) \psi(s, x) d s, \\
& F(t, \psi, \tilde{B} \psi)(x)=\int_{-\infty}^{0} b_{2}(t, s, x, \psi(s, x)) d s+\tilde{B} \psi(x),
\end{aligned}
$$

where

$$
\tilde{B} \psi(x)=\int_{0}^{t} \int_{-\infty}^{0} b_{3}(t) b_{4}(s, \tau-s, \psi(\tau, x)) d \tau d s .
$$

Also define the maps $I_{k}$ and $B$ by

$$
\begin{aligned}
& I_{k}(\psi)(x)=\int_{-\infty}^{0} \eta_{k}(s) \psi(s, x) d s, \\
& (B u)(t)(x)=\tilde{u}(t, x) .
\end{aligned}
$$

Using these definitions, we can represent system (5.1)-(5.5) in the abstract form (1.1)(1.3). Moreover, $G, I_{k}$ are bounded linear operators on $\mathcal{B}$ with $\|G\| \leq L_{G}$ and $\left\|I_{k}\right\| \leq L_{k}$, $k=1,2, \ldots, m$. Using (ii), we see that $F$ is continuous and $\|F\|_{L(\mathcal{B}, H)} \leq L_{F}$, where $L_{F}=$ $\widetilde{L}_{1}\left\|a_{1}\right\|_{\infty}+b \widetilde{L}_{2}\left\|b_{3}\right\|_{\infty}\left\|a_{3}\right\|_{\infty}$. Further, we can impose some suitable conditions on the above-defined functions to verify the assumptions on Theorem 3.1. Therefore, assumptions (H1)-(H6) and (B1) all hold; the associated linear system of (5.1)-(5.5) is not exactly 
controllable but it is approximately controllable. Hence by Theorems 3.1 and 4.1, system (5.1)-(5.5) is approximately controllable on $[0, b]$.

\section{Competing interests}

The authors declare that they have no competing interests.

\section{Authors' contributions}

The authors completed the paper, and read and approved the final manuscript.

\section{Acknowledgements}

The authors would like to thank the editor and the reviewers for their constructive comments and suggestions. This work is supported by the National Natural Science Foundation of China (Grant No. 11461019) and is supported by the President Found of Scientific Research Innovation and Application of Hexi University (Grant No. xz2013-10).

\section{Received: 19 September 2014 Accepted: 14 April 2015 Published online: 28 April 2015}

\section{References}

1. Benchohra, M, Henderson, J, Ntouyas, SK: Impulsive Differential Equations and Inclusions. Hindawi Publishing Corporation, New York (2006)

2. Haddad, WM, Chellabonia, V, Nersesov, SG, Sergey, G: Impulsive and Hybrid Dynamical Systems: Stability, Dissipativity, and Control. Princeton University Press, Princeton (2006)

3. Hernández, E, Pierri, M, Goncalves, G: Existence results for an impulsive abstract partial differential equation with state-dependent delay. Comput. Math. Appl. 52, 411-420 (2006)

4. Kilbas, AA, Srivastava, HM, Trujillo, JJ: Theory and Applications of Fractional Differential Equations. North-Holland Mathematics Studies, vol. 204. Elsevier, Amsterdam (2006)

5. Lakshmikantham, V: Theory of fractional differential equations. Nonlinear Anal. 60, 3337-3343 (2008)

6. Lakshmikantham, V, Vatsala, AS: Basic theory of fractional differential equations. Nonlinear Anal. 69, 2677-2682 (2008)

7. El-Borai, MM: Some probability densities and fundamental solutions of fractional evolution equations. Chaos Solitons Fractals 14, 433-440 (2002)

8. El-Borai, MM: Semigroups and some nonlinear fractional differential equations. Appl. Math. Comput. 149, 823-831 (2004)

9. Yan, Z: Existence results for fractional functional integrodifferential equations with nonlocal conditions in Banach spaces. Ann. Pol. Math. 97, 285-299 (2010)

10. Zhou, Y, Jiao, F: Existence of mild solutions for fractional neutral evolution equations. Comput. Math. Appl. 59 1063-1077 (2010)

11. Debbouche, A, Nieto, JJ: Sobolev type fractional abstract evolution equations with nonlocal conditions and optimal multi-controls. Appl. Math. Comput. 245, 74-85 (2014)

12. Debbouche, A, Torres, DFM: Sobolev type fractional dynamic equations and optimal multi-integral controls with fractional nonlocal conditions. Fract. Calc. Appl. Anal. 18, 95-121 (2015)

13. Santos, JPC, Arjunan, MM, Cuevas, C: Existence results for fractional neutral integro-differential equations with state-dependent delay. Comput. Math. Appl. 62, 1275-1283 (2011)

14. Agarwal, RP, de Andrade, B, Siracusa, G: On fractional integro-differential equations with state-dependent delay. Comput. Math. Appl. 62, 1143-1149 (2011)

15. de Andrade, B, Santos, JPC: Existence of solutions for a fractional neutral integro-differential equation with unbounded delay. Electron. J. Differ. Equ. 2012, 90 (2012)

16. Mophou, GM: Existence and uniqueness of mild solutions to impulsive fractional differential equations. Nonlinear Anal. 72, 1604-1615 (2010)

17. Shu, $X-B$, Lai, $Y, C$ Chen, $Y$ : The existence of mild solutions for impulsive fractional partial differential equations. Nonlinear Anal. 74, 2003-2011 (2011)

18. Dabas, J, Chauhan, A: Existence and uniqueness of mild solution for an impulsive neutral fractional integro-differential equation with infinite delay. Math. Comput. Model. 57, 754-763 (2013)

19. Balachandran, K, Kiruthika, S, Trujillo, JJ: Existence results for fractional impulsive integrodifferential equations in Banach spaces. Commun. Nonlinear Sci. Numer. Simul. 16, 1970-1977 (2011)

20. Balachandran, K, Park, JY: Controllability of fractional integrodifferential systems in Banach spaces. Nonlinear Anal. Hybrid Syst. 3, 363-367 (2009)

21. Wang, J, Zhou, Y: Complete controllability of fractional evolution systems. Commun. Nonlinear Sci. Numer. Simul. 17, 4346-4355 (2012)

22. Sakthivel, R, Mahmudov, NI, Nieto, JJ: Controllability for a class of fractional-order neutral evolution control systems. Appl. Math. Comput. 218, 10334-10340 (2012)

23. Yan, Z: Controllability of fractional-order partial neutral functional integrodifferential inclusions with infinite delay. J. Franklin Inst. 348, 2156-2173 (2011)

24. Tai, Z, Wang, X: Controllability of fractional-order impulsive neutral functional infinite delay integrodifferential systems in Banach spaces. Appl. Math. Lett. 22, 1760-1765 (2009)

25. Debbouchea, A, Baleanu, D: Controllability of fractional evolution nonlocal impulsive quasilinear delay integro-differential systems. Comput. Math. Appl. 62, 1442-1450 (2011)

26. Liu, Z, Li, X: On the controllability of impulsive fractional evolution inclusions in Banach spaces. J. Optim. Theory Appl. 156, 167-182 (2013)

27. Triggiani, R: A note on the lack of exact controllability for mild solutions in Banach spaces. SIAM J. Control Optim. 15 407-411 (1977)

28. Mahmudov, NI, Denker, A: On controllability of linear stochastic systems. Int. J. Control 73, 144-151 (2000) 
29. Dauer, JP, Mahmudov, NI: Approximate controllability of semilinear functional equations in Hilbert spaces. J. Math. Anal. Appl. 273, 310-327 (2002)

30. Sakthivel, R, Ren, Y, Mahmudov, NI: On the approximate controllability of semilinear fractional differential systems. Comput. Math. Appl. 62, 1451-1459 (2011)

31. Sakthivel, R, Ren, Y: Approximate controllability of fractional differential equations with state-dependent delay. Results Math. 63, 949-963 (2013)

32. Sakthivel, R, Suganya, S, Anthoni, SM: Approximate controllability of fractional stochastic evolution equations. Comput. Math. Appl. 63, 660-668 (2012)

33. Kumar, S, Sukavanam, N: Approximate controllability of fractional order semilinear systems with bounded delay J. Differ. Equ. 252, 6163-6174 (2012)

34. Sukavanam, N, Kumar, S: Approximate controllability of fractional order semilinear delay systems. J. Optim. Theory Appl. 151, 373-384 (2011)

35. Yan, Z: Approximate controllability of partial neutral functional differential systems of fractional order with state-dependent delay. Int. J. Control 85, 1051-1062 (2012)

36. Debbouche, A, Torres, DFM: Approximate controllability of fractional nonlocal delay semilinear systems in Hilbert spaces. Int. J. Control 86, 1577-1585 (2013)

37. Debbouche, A, Torres, DFM: Approximate controllability of fractional delay dynamic inclusions with nonlocal control conditions. Appl. Math. Comput. 243, 161-175 (2014)

38. Benchohra, M, Litimein, S, N'Guérékata, G: On fractional integro-differential inclusions with state-dependent delay in Banach spaces. Appl. Anal. 92, 33-35 (2013)

39. Yan, Z: On a nonlocal problem for fractional integrodifferential inclusions in Banach spaces. Ann. Pol. Math. 101 87-103 (2011)

40. O'Regan, D: Nonlinear alternatives for multivalued maps with applications to operator inclusions in abstract spaces. Proc. Am. Math. Soc. 127(12), 3557-3564 (1999)

41. Yosida, K: Functional Analysis, 6th edn. Springer, Berlin (1980)

42. Fitzpatrick, PM, Petryshyn, WV: Fixed point theorems for multivalued noncompact acyclic mappings. Pac. J. Math. 54, 17-23 (1974)

43. Hale, JK, Kato, J: Phase spaces for retarded equations with infinite delay. Funkc. Ekvacioj 21, 11-41 (1978)

44. Lasota, A, Opial, Z: An application of the Kakutani-Ky Fan theorem in the theory of ordinary differential equations. Bull. Acad. Pol. Sci., Sér. Sci. Math. Astron. Phys. 13, 781-786 (1965)

45. Hino, Y, Murakami, S, Naito, T: Functional-Differential Equations with Infinite Delay. Lecture Notes in Mathematics, vol. 1473. Springer, Berlin (1991)

\section{Submit your manuscript to a SpringerOpen ${ }^{\circ}$ journal and benefit from:}

- Convenient online submission

Rigorous peer review

Immediate publication on acceptance

- Open access: articles freely available online

- High visibility within the field

- Retaining the copyright to your article 